OPEN ACCESS

Edited by:

Jagadeesh Bayry,

Institut National de la Santé et de

la Recherche Médicale, France

Reviewed by:

Junji Xing,

Houston Methodist Research

Institute, United States

Ashley Mansell,

Hudson Institute of Medical

Research, Australia

*Correspondence:

Zhen F. Fu

zf123@yahoo.com;

Ling Zhao

zling604@yahoo.com

Specialty section: This article was submitted to

Molecular Innate Immunity,

a section of the journal

Frontiers in Immunology

Received: 16 September 2017 Accepted: 27 December 2017

Published: 19 January 2018

Citation:

Tian B, Zhou M, Yang Y, Yu L, Luo Z, Tian D, Wang K, Cui M, Chen H,

Fu ZF and Zhao L (2018) LabAttenuated Rabies Virus Causes

Abortive Infection and Induces

Cytokine Expression in Astrocytes by Activating Mitochondrial Antiviral-

Signaling Protein Signaling Pathway.

Front. Immunol. 8:2011.

doi: 10.3389/fimmu.2017.02011

\section{Lab-Attenuated Rabies Virus Causes Abortive Infection and Induces Cytokine Expression in Astrocytes by Activating Mitochondrial Antiviral- Signaling Protein Signaling Pathway}

Bin Tian 1,2, Ming Zhou 1,2, Yu Yang ${ }^{1,2}$, Lan Yu ${ }^{1,2}$, Zhaochen Luo 1,2, Dayong Tian ${ }^{1,2}$, Ke Wang ${ }^{1,2}$, Min Cui ${ }^{1,3}$, Huanchun Chen ${ }^{1,2,3}$, Zhen F. Fu ${ }^{1,3,4 *}$ and Ling Zhao ${ }^{1,2,3 *}$

'State Key Laboratory of Agricultural Microbiology, Huazhong Agricultural University, Wuhan, China, ${ }^{2}$ Key Laboratory of Preventive Veterinary Medicine of Hubei Province, Huazhong Agricultural University, Wuhan, China, ${ }^{3}$ College of Veterinary Medicine, Huazhong Agricultural University, Wuhan, China, ${ }^{4}$ Department of Pathology, University of Georgia, Athens, GA, United States

Rabies is an ancient disease but remains endemic in most parts of the world and causes approximately 59,000 deaths annually. The mechanism through which the causative agent, rabies virus (RABV), evades the host immune response and infects the host central nervous system (CNS) has not been completely elucidated thus far. Our previous studies have shown that lab-attenuated, but not wild-type (wt), RABV activates the innate immune response in the mouse and dog models. In this present study, we demonstrate that lab-attenuated RABV causes abortive infection in astrocytes, the most abundant glial cells in the CNS. Furthermore, we found that lab-attenuated RABV produces more double-stranded RNA (dsRNA) than wt RABV, which is recognized by retinoic acidinducible gene I (RIG-I) or melanoma differentiation-associated protein 5 (MDA5). Activation of mitochondrial antiviral-signaling protein (MAVS), the common adaptor molecule for RIG-I and MDA5, results in the production of type I interferon (IFN) and the expression of hundreds of IFN-stimulated genes, which suppress RABV replication and spread in astrocytes. Notably, lab-attenuated RABV replicates in a manner identical to that of wt RABV in MAVS-/- astrocytes. It was also found that lab-attenuated, but not $w t, R A B V$ induces the expression of inflammatory cytokines via the MAVS- p38/NF-kB signaling pathway. These inflammatory cytokines increase the blood-brain barrier permeability and thus enable immune cells and antibodies infiltrate the CNS parenchyma, resulting in RABV control and elimination. In contrast, wt RABV restricts dsRNA production and thus evades innate recognition by RIG-I/MDA5 in astrocytes, which could be one of the mechanisms by which wt RABV evades the host immune response in resident CNS cells. Our findings suggest that astrocytes play a critical role in limiting the replication of lab-attenuated RABV in the CNS.

Keywords: rabies virus, astrocytes, double-strand RNA, cytokine expression, mitochondrial antiviral-signaling protein signaling pathway 


\section{INTRODUCTION}

Rabies is an acute encephalomyelitis. The hallmark of rabies is that the disease is almost always fatal once clinical signs develop $(1,2)$. The causative agent, rabies virus (RABV), is a negative-strand RNA virus belonging to the genus Lyssavirus in the family Rhabdoviridae (3). RABV enters neurons from the neuromuscular junction closest to the site of infection. After a short incubation, RABV travels to the central nervous system (CNS) through sensory or motor neurons. Only slight tissue damage and neuroinflammation can be observed in the brains of rabid patients (4). In contrast, lab-attenuated RABV induces extensive inflammation and apoptosis, as well as increases in the expression levels of innate immunity-related genes in the CNS of infected mice (5-10). These findings suggest that wt, but not lab-attenuated, RABV evades the host immune responses.

The innate immune system is the first line of defense against viral invasion. Viruses are usually confronted by various pattern recognition receptors (PRRs), including Toll-like receptors (TLRs) and retinoic acid-inducible gene I (RIG-I) like helicases (RLRs) (11). TLR family members, such as TLR3 and TLR7, are generally involved in recognizing negative-strand RNA viruses (12). TLR3 binds double-stranded RNA (dsRNA), whereas TLR7 recognizes single-strand RNA. The main sources of dsRNA in infections with single-strand RNA viruses are the replicative intermediates generated by viral RNA-dependent RNA polymerase (13). TLR3 and TLR7 initiate signaling though the adaptor molecules TRIF and MyD88, respectively. Both signaling cascades triggered by these proteins lead to IRF3 phosphorylation in the $\mathrm{C}$ terminal region at serine 386 , which is critical for IRF3 activation by two IкB kinases (TBK-1 and IKK $)(14,15)$. Activated IRF3 homo- or hetero-dimerizes with IRF7 and then translocates into the nucleus, to interacts with the CREB binding protein $\mathrm{CBP} / \mathrm{p} 300$ and stimulates the transcription of interferon (IFN) $\beta$, as well as some IFN-stimulated genes (ISGs) $(15,16)$. Alternatively, RNA viruses can be recognized by two RLRs, RIG-I, and melanoma differentiation-associated protein 5 (MDA5), located in the cytoplasm $(17,18)$. RIG-I solely senses short and blunt dsRNA of negative-strand RNA viruses containing $5^{\prime}$ triphosphate RNA in the panhandle region of their single-stranded genome (17). Unlike RIG-I, MDA5 preferentially binds blunt-ended dsRNA, with or without 5 ' triphosphate (18). RIG-I and MDA5 signaling is mediated through mitochondrial antiviral-signaling protein (MAVS), which is also known as IPS1, VISA or Cardif (19). Similar to TLR signaling, RLR signaling results in IRF3 activation and nuclear translocation (20).

Several groups have attempted to identify the PRRs that recognize RABV. Prehaud et al. found that TLR3 mRNA expression is upregulated following RABV infection in postmitotic human neurons (21). Furthermore, enhanced TLR3 expression has also been observed in the cerebellar cortical tissues of rabies patients (22). The observation that TLR3 is upregulated following infection suggests that TLR3 plays a role in the innate recognition of RABV. Faul et al. found that both RIG-I and MDA5 were responsible for inducing dendritic cell (DC) activation and type I IFN production upon RABV infection (23). MAVS, the adaptor protein for both RIG-I and MDA5, is essential for inducing innate immune responses in DCs. In another study, Li et al. found that mice lacking TLR7 exhibited a phenotype associated with intermediate mortality rates between those of MyD88-/- and control mice, indicating that TLR7 may play an important role in controlling RABV infections. However, the role of TLR7 in RABV infection is not entirely understood (24).

Astrocytes are the most abundant glial cells in the CNS and constitute the blood-brain barrier (BBB) along with endothelial cells and pericytes (25). Astrocytes are generally involved in regulating the CNS microenvironment and also play roles in neuronal metabolic support, synaptic transmission, and neurotropism. Moreover, astrocytes participate in developing and maintaining the $\mathrm{BBB}$ and guiding neuronal migration during development. The roles of astrocytes in innate immunity and inflammation have been reported recently (26). Astrocytes are the reservoir for many neuroinvasive viruses, such as human immunodeficiency virus, Theiler's murine encephalomyelitis virus (TMEV), John Cunningham virus, and herpes simplex virus (HSV) (27-30). Astrocytes have also been shown to play multiple roles in viral infections. Specifically, they can increase BBB permeability (31) by producing cytokines and degrading tight junction proteins (32). Moreover, astrocytes have been shown to induce the innate immune response to produce IFN (33). An early report showed that both wt and lab-attenuated RABV could successfully infect primary astrocytes in the early stages of RABV infection (34). A recent study demonstrated that a RABV vaccine strain SAD-L16 caused an abortive infection in astrocytes, but the detailed mechanism was not revealed (35). In this study, it was found that lab-attenuated RABV produces higher level of dsRNA than wt RABV, which is recognized by RIG-I/MDA5 and results in the activation of MAVS signaling pathway. Following MAVS activation, the production of ISG and inflammatory cytokines helps to clear the lab-attenuated RABV from the CNS. In contrast, wt RABV can maintain a persistent infection in astrocytes by evading the innate recognition.

\section{MATERIALS AND METHODS}

\section{Cells, Viruses, Antibodies, and Mice}

Mouse bEnd.3 cells and Vero cells were obtained from the American Type Culture Collection (ATCC; Manassas, VA, USA) and were maintained in Dulbecco's modified Eagle medium (DMEM) supplemented with fetal bovine serum (FBS; Gibco, Carlsbad, CA, USA). Mouse neuroblastoma (NA) cells were maintained in RPMI-1640 medium (Thermo-Fisher, USA) supplemented with $10 \%$ FBS.

The DRV-AH08 (DRV) was isolated from a rabid dog in Anhui province, China (36, 37). CVS-B2c (B2c), originated from CVS-24 virus by passage in BHK-21 cells (6), has been used as a lab-attenuated RABV (38-40). Both DRV and CVS-B2c were 
propagated in suckling ICR mouse brains. All the viruses were manipulated under the standard biosecurity procedures made by The Ministry of Agriculture of China.

A rabbit anti-ubiquitin protein $\mathrm{C}$ (UBC) polyconal antibody was purchased from ABclonal Technology (Woburn, MA, USA). A rabbit anti-RIG-I monoclonal antibody was purchased from Enzo Life Technology (Farmingdale, NY, USA), and rabbit polyclonal antibodies against IRF7, STAT1 and Occludin were obtained from Santa Cruz Biotechnology (Santa Cruz, CA, USA). A rabbit antiphospho-IRF7 monoclonal antibody was purchased from Cell Signaling Technology (Washington, DC, USA), and rabbit anti-IFIT1 and IRF3 monoclonal antibodies were purchased from Abcam (Cambridge, MA, USA). A rabbit polyclonal anti-claudin-5 antibody, a biotinylated goat anti-rabbit or mouse 594 antibody, and an Alexa Fluor 488-conjugated goat antimouse or rabbit antibody were purchased from Invitrogen (Grand Island, NY, USA). Mouse monoclonal anti-Zoluna occludens-1 (anti-ZO-1) antibodies were obtained from Sigma (St. Louis, MO, USA), and mouse monoclonal anti-GAPDH antibody was purchased from ProteinTech (Wuhan, China). The mouse anti-RABV N and $\mathrm{P}$ monoclonal antibodies were prepared in our laboratory, and the fluorescein isothiocyanate (FITC)-conjugated antiRABV nucleoprotein antibody used herein was obtained from Fujirebio (Malvern, PA, USA).

Female C57BL/6 mice (6-8 weeks old) were purchased from the Center for Disease Control of Hubei province (Wuhan, China), and MAVS-knockout (MAVS-/-, Stock No: 008634-B6; 129-Mavs ${ }^{\text {tmlZjc } / J, ~ U S A) ~ a n d ~ T L R 7-k n o c k o u t ~(T L R 7-/-, ~ S t o c k ~ N o: ~}$ 008380-B6; 129S1-Tlr ${ }^{\text {tm1Flv }} /$ J, USA) mice were purchased from the Jackson Laboratory (Bar Harbor, ME, USA). All the mice were housed in the animal facility at Huazhong Agricultural University, Wuhan, China.

\section{Mouse Infections}

Female wt or MAVS-knockout (MAVS-/-) C57BL/6 mice (6-8 weeks old) were infected intracerebally (i.c.) with $20 \mu \mathrm{L}$ of DRV-AH08 (200 FFU), B2c (20 FFU), or mock infected with the same volume of DMEM. At 7 days postinfection (d.p.i.), the mice were euthanized with $\mathrm{CO}_{2}$ when moribund, and their brains were collected for immunohistochemistry analysis.

\section{BBB Permeability Assay}

Blood-brain barrier permeability was determined by measuring sodium fluorescein $(\mathrm{NaF})$ uptake as described previously with minor modifications (41). Briefly, $100 \mu \mathrm{l}$ of $\mathrm{NaF}(100 \mathrm{mg} / \mathrm{ml})$ was injected intraperitoneally (i.p.) into each mouse. After anesthetization, peripheral blood and brains of each mouse were collected. The fluorescence in serum and brain homogenate samples was analyzed by a spectrophotometer (BioTek Instruments, VT, USA) with excitation at $485 \mathrm{~nm}$ and emission at $530 \mathrm{~nm}$. Standards (125-4,000 g/ml) were prepared to calculate the $\mathrm{NaF}$ content. NaF uptake into tissue is calculated as ( $\mu \mathrm{g}$ of fluorescence spinal cord $/ \mathrm{mg}$ of tissue)/( $\mu$ g of fluorescence sera $/ \mathrm{ml}$ of blood) to normalize values for blood levels of the dye at the time of tissue collection.

\section{Immunohistochemistry}

To detect $\mathrm{CD} 45^{+}$cell in the brain, infected mice were anesthetized with ketamine-xylazine $(0.1 \mathrm{ml} / 10 \mathrm{~g}$ body weight $)$, perfused with $50 \mathrm{ml} \mathrm{PBS}$, and then the brains were transferred into $4 \%$ neutral buffered paraformaldehyde (PFA) for at least $24 \mathrm{~h}$ (39). Briefly, the brain sections were antigenic recovered and blocked with donkey serum, incubated with primary antibodies overnight at $4^{\circ} \mathrm{C}$, and then secondary antibodies was applied. PBS was treated as a negative control by replacing primary antibodies. Sections were photographed and analyzed using an Olympus BX41 microscope (Tokyo, Japan).

\section{Primary Astrocyte Isolation}

Primary mixed glial cell cultures were established as described previously (42). Briefly, the brain cells of 1- to 3-day-old neonatal C57BL/6 mice were dissociated by repeated pipetting and then passed through a 75-nm nylon mesh (Corning, NY, USA). The cells were subsequently washed once in cold PBS and cultured in DMEM (with high glucose) supplemented with 10\% FBS and 1\% penicillin-streptomycin. The medium was changed on days 3, 5, and 7 for the astrocytes and on day 3 only for the microglia. On day 10 , the flasks were shaken at $260 \mathrm{rpm}$ for $2 \mathrm{~h}$ to remove any non-adherent cells (mainly microglia). The remaining adherent astrocytes were detached with trypsin-EDTA and then plated again for further experiments. The purity of the astrocyte cultures was greater than $95 \%$.

\section{Neuron Isolation}

Mouse neurons were obtained from embryonic mouse brains as previously described (42) and then dissociated by repeated pipetting (approximately 20 times) before being passed through a 75-nm nylon mesh. The cells were then washed once in cold PBS and cultured in DMEM (with high glucose) supplemented with $5 \%$ FBS and $1 \%$ penicillin-streptomycin for $6 \mathrm{~h}$, after which the medium was replaced with serum-free neural-basal medium (Invitrogen, Carlsbad, CA, USA) supplemented with 2\% B-27 (Invitrogen, Carlsbad, CA, USA).

\section{Virus Titration}

Viral titers were determined by direct fluorescent antibody assay (40). NA cells cultured in 96-well plates were inoculated with viruses diluted serial 10 -fold and then incubated at $37^{\circ} \mathrm{C}$ for $48 \mathrm{~h}$. Then the culture supernatant was subsequently removed, and the cells were fixed and stained with FITC-conjugated antiRABV $\mathrm{N}$ antibodies. The antigen-positive foci were counted under a fluorescence microscope (Zeiss, Germany), and the viral titers were calculated as FFU per milliliter. All titrations were conducted in quadruplicate.

\section{Confocal Microscopy}

Primary astrocytes, neurons or bEnd.3 cells were seeded on coverslips and allowed to grow until they formed a confluent monolayer, after which they were infected with RABV or suspended in medium containing the supernatants of infected astrocytes. The cells were subsequently fixed with $4 \%$ PFA, permeabilized with $0.1 \%$ Triton X-100 and then stained with 
antibodies against dsRNA, RABV N, RABV P, Claudin-5, Occludin, ZO-1, or DAPI.

Infected wt or MAVS-/- mice were anesthetized with ketamine-xylazine $(0.1 \mathrm{~mL} / 10 \mathrm{~g}$ body weight $)$ and then perfused with PBS followed by $10 \%$ neutral buffered formalin, as described previously (39). Three independent mouse brain samples were collected from each group and embedded in paraffin for coronal sectioning. The sections were subsequently stained with antibodies against GFAP, MAP2, RABV P, RABV $\mathrm{N}$, or DAPI. After being washed, the cells or sections were incubated with an Alexa Fluor 488-conjugated goat antirabbit or mouse secondary antibodies or an Alexa Fluor 594-conjugated goat antirabbit or mouse secondary antibodies for $1 \mathrm{~h}$ at room temperature. Staining was visualized with a Nikon A1 confocal laser microscope system equipped with NIS-Elements imaging software (Nikon, Melville, NY, USA) and was quantified using Fiji, an ImageJ distribution package manufactured by NIH (http://imagej.net/Introduction). Mean fluorescence intensity (MFI) was quantified using the region of interest, which encompassed the entire cell to include the membrane, and background staining was quantified using three negatively stained regions per cell. These regions were subtracted from the total MFI.

\section{Inhibitor Treatment}

For the protein synthesis inhibition tests, primary astrocytes were pretreated with cycloheximide (CHX) (Invivogen, San Diego, CA, USA) for $1 \mathrm{~h}$ at a dose of $50 \mu \mathrm{g} / \mathrm{mL}$, infected with DRV or $\mathrm{B} 2 \mathrm{c}$ at an MOI of 0.1 and then continued to be incubated with $\mathrm{CHX}$ for another $24 \mathrm{~h}$. For the TBK1 activation blockage assays, BX795 (Invivogen, San Diego, CA, USA) at a dose of $1 \mu \mathrm{M}$ was used to treat primary astrocytes.

For the inflammatory pathway blockage assays, the primary astrocytes were pretreated with a p38 inhibitor (Skepinone-L; SELLEK, Houston, TX, USA), a JNK inhibitor (JNK inhibitor IX; SELLEK, Houston, TX, USA), an NF-кB inhibitor (sc75741; SELLEK, Houston, TX, USA), or DMSO as control at a dose of $5 \mu \mathrm{g} / \mathrm{mL}$ for $1 \mathrm{~h}$. Then the cells were infected with DRV or B2c at MOI 1 and incubated with the above inhibitors for $48 \mathrm{~h}$.

\section{ELISA}

The concentrations of TNF- $\alpha$ and IL- 6 in the supernatant of astrocytes were measured by the commercial ELISA kits according to the manufacturer's instruction (ABclonal Technology, Woburn, MA, USA).

\section{qRT-PCR}

RNA was isolated with TRIzol ${ }^{\circledR}$ Reagent (Invitrogen), according to the manufacturer's instructions, and qRT-PCR was performed as described previously (39). Briefly, $800 \mathrm{ng}$ of total RNA (from either cells or tissue) was transcribed into cDNA in a reaction mixture with a total of volume of $20 \mu \mathrm{L}$ using a Superscript III Reverse Transcription Kit (Toyobo). The reaction mixture comprised $1 \mu \mathrm{L}$ of cDNA combined with $5 \mu \mathrm{L}$ of iQ 5 SYBR Green Mix (BioRad, Hercules, CA, USA), $3 \mu \mathrm{L}$ of diethyl pyrocarbonatetreated water, and $0.5 \mu \mathrm{L}$ of primer mix (the concentration of each primer was $10 \mathrm{mM}$ ). The cDNA was amplified using an
iQ5 iCycler (Bio-Rad), and the cycle threshold (CT) values were recorded. The $C T$ value was inversely correlated with the mRNA concentration, and each $C T$ unit represented a twofold change in the mRNA concentration. Basal mRNA expression levels were expressed as ${ }^{\Delta} C T$ values and were normalized to $\beta$-actin mRNA expression levels $\left[{ }^{\Delta} C T C T\right.$ (ISG) $/{ }^{\Delta} C T$ ( $\beta$-actin) $]$. Induced mRNA expression levels were expressed as fold changes relative to mock-infection levels using the $2^{\triangle \Delta C T}$ method. All the primer sequences are listed in (Table 1). To quantify cellular RABV N RNA levels, we transcribed the total RNA using Avian myeloblastosis virus Reverse Transcriptase XL (TAKARA, Kusatsu, Japan) and a primer specific for the RABV N genomic sequence. A standard curve was generated from serially diluted plasmids carrying a RABV N gene and the copy numbers of $\mathrm{N}$ mRNA were normalized to $1 \mathrm{mg}$ of total RNA.

\section{Western Blot}

The cells were lysed with RIPA buffer containing protease inhibitors (Roche), and the protein concentrations were measured using a DC protein assay kit (Bio-Rad). Equal quantities of protein were resolved by 12 or $15 \%$ SDS-PAGE and then transferred to polyvinylidene difluoride membranes (Bio-Rad), which were blocked with $5 \%$ nonfat milk before being incubated with primary antibodies against RIG-I, phosphorylated IRF7 (p-IRF7), STAT1, IFIT1, RABV N, Claudin-5, Occludin, ZO-1, or GAPDH and then probed with the appropriate secondary antibodies. The blots were then visualized using ECL reagent (GE, Pittsburgh, PA, USA) and detected under an Intelligent dark box II (GE, Pittsburgh, PA, USA).

TABLE 1 | Primers used for quantification of viral mRNA, IFN-stimulated genes, chemokines, and cytokines.

\begin{tabular}{lll}
\hline Primer name & Sequence $\left(\mathbf{5}^{\prime} \mathbf{- 3} \mathbf{3}^{\prime}\right)$ & Use \\
\hline $\begin{array}{lll}\text { Retinoic acid-inducible } \\
\text { gene I (RIG-I) F }\end{array}$ & GCGTCTCAGTGCAGCACATCATT & qRT-PCR \\
RIG-I R & GGGTCCCGTGACTCTCCAAGTTT & qRT-PCR \\
$\begin{array}{l}\text { Melanoma } \\
\text { differentiation- }\end{array}$ & GCTGCTAAAGACGGAAATCG & qRT-PCR
\end{tabular}

associated protein 5

(MDA5) F

MDA5 R

Mitochondrial

CTTGTCGCTGTCATTGAGGA

qRT-PCR

antiviral-signaling

protein (MAVS) F

MAVS R

IRF7 F

IRF7 R

Interferon (IFN)- $\beta$ F

IFN- $\beta$ R

STAT1 $F$

STAT1 R

IFIT1 F

IFIT1 R

Rabies virus

ATGCCGTITGCTGAAGAC

qRT-PCR

CTAGTGCAGACGCCGCCG

CTGGAGCCATGGGTATGCA

AAGCACAAGCCGAGACTGCT

CACAGCCCTCTCCATCAAC

GCATCTTCTCCGTCATCTCC

GTGGACATTGAGTTCTTGGTGAGATCC

CCTICTCATTCTGTCGACTTGTTGG

GCATCACCTTCCTCTGGCTA

TGTTGTTCAGTGCCTTCTGG

ACACCGGCAACTACAAGACA

(RABV) NF

RABV NR

$\beta$-actin F

$\beta$-actin R
ATGGTACTCCAGTTGGCACA AGGTGACAGCATTGCTTCTG GCTGCCTCAACACCTCAAC
qRT-PCR qRT-PCR qRT-PCR qRT-PCR qRT-PCR qRT-PCR qRT-PCR qRT-PCR qRT-PCR qRT-PCR and CDNA synthesis qRT-PCR qRT-PCR qRT-PCR 


\section{Cytokine and Chemokine Protein Quantification}

Primary astrocytes were infected with DRV or B2c at an MOI of 1 or mock infected, and the cell supernatants were collected at 24,48 , or $72 \mathrm{~h}$ p.i. The levels of several inflammatory cytokines, including TNF- $\alpha$, IL-6, IL-1 $\beta$, IL-17, IFN- $\gamma$, and vascular endothelial growth factor (VEGF), were quantified in the mockor RABV-infected cell supernatants by a Quantibody Mouse Cytokine Array 1 Kit (RayBiotech, Norcross, GA, USA), according to the manufacturer's protocol. The array was scanned using a GenePix 4000B (Molecular Devices, Axon Instruments, Silicon Valley, CA, USA), and the data were collected at several PMT values ranging from 540 to 790 gain using GenePix Pro-software. The 590-gain PMT scan generated the optimal standard curve, and the results of this scan were analyzed using Q-Analyzer Software for QAM-CYT-1 (RayBiotech).

\section{Transendothelial Permeability Assay}

Transendothelial permeability assay was performed according to the methods described previously (43), with some modifications. B.END3 cells were grown on $3-\mu \mathrm{m}$-pore transwell filter inserts until they reached $100 \%$ confluency. The medium was then treated with UV-inactivated cell culture supernatants collected from RABV-infected astrocytes. After the cells had incubated for $48 \mathrm{~h}$, they were treated apically with Dextran-FITC at a dose of $0.1 \mu \mathrm{g} / \mathrm{mL}$ for $30 \mathrm{~min}$. The samples were then removed from the lower chamber and subjected to fluorescence measurement, which were performed using a fluorimeter (BioTek, Winooski,
VT, USA; the excitation wavelength was $492 \mathrm{~nm}$, and the emission wavelength was $520 \mathrm{~nm}$ ). The fluorescence values for the experimental cells were subsequently compared to corresponding values for a control cell monolayer.

\section{Statistical Analysis}

Data are expressed as the mean and standard error of the mean (SEM), and the significance of the differences between groups was evaluated by Student's $t$ test or one-way analysis of variance followed by Tukey's post hoc test. The survival ratios were analyzed by Log-rank (Mantel-Cox) test. The asterisks indicated statistical significance $\left({ }^{*}, P<0.05 ;{ }^{* *}, P<0.01 ;{ }^{* *}, P<0.001\right)$. Graphs were plotted and analyzed using GraphPad Prism software, version 6.0 (GraphPad Software, La Jolla, CA, USA).

\section{RESULTS}

\section{Comparison of the Pathogenicity of DRV-AH08 and CVS-B2c in Mice}

First, the pathogenicity of the wt RABV strain DRV-AH08 (DRV) and lab-attenuated strain CVS-B2c (B2c) used in this study were compared in a mouse model. C57/BL6 mice were i.c. inoculated with 20 FFU B2c or DRV and the development of rabies was observed. As expected, DRV-infected mice displayed development of the diseases at 5 d.p.i. and all moribund at 9 d.p.i., earlier than B2c-infected mice which all succumb to rabies at 10 d.p.i. (Figure 1A).

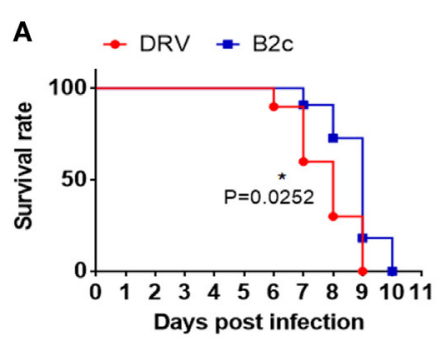

D

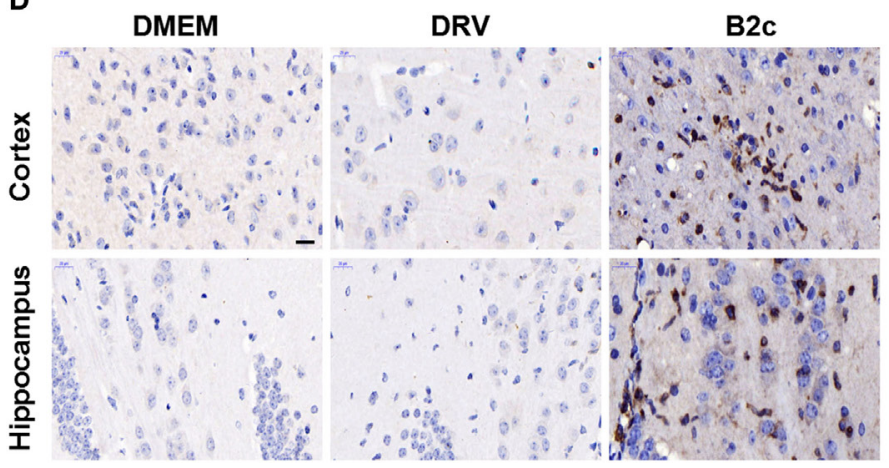

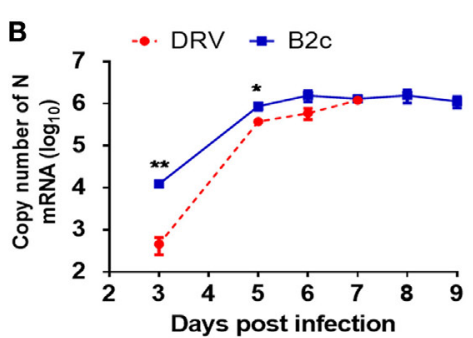

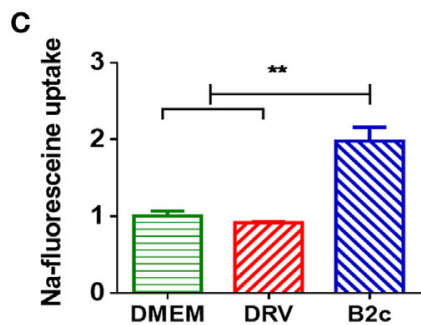

E

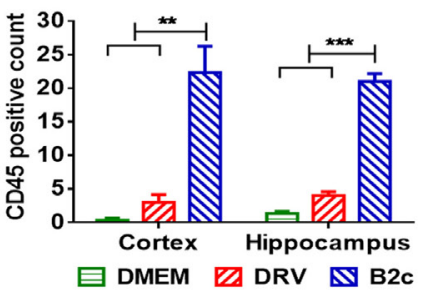

FIGURE 1 Wt and lab-attenuated rabies virus infection in mice. Groups of C57BL/6 mice $(n=10)$ were intracerebally (i.c.) inoculated with $20 \mathrm{FFU}$ DRV or B2c, and observed daily for 2 weeks. (A) The survival ratio was presented and analyzed by Log-rank (Mantel-Cox) test. (B) Groups of C57BL/6 mice ( $n=5)$ were i.c. infected with 200 FFU DRV or 20 FFU B2c. At indicated time points p.i., N mRNA of DRV and B2c in the mouse brain was analyzed by qRT-PCR. (C) The change of blood-brain barrier permeability in the DRV-, B2c-, and mock-infected mouse brain was determined by measuring the Na-fluorescence uptake. (D) The inflammation in DRV-, B2C-, and mock-infected mouse brains was analyzed by immunohistochemistry using anti-CD45 antibody. The scale bars represent $20 \mu \mathrm{m}$. (E) The inflammation was quantified by counting CD45+ lymphocytes from at least three different randomly selected areas. 
We found that $\mathrm{B} 2 \mathrm{c}$ replicated faster than DRV at the early stage of the infection in the CNS. To ensure that the viral load in the brains is similar, C57/BL6 mice were i.c. inoculated with $20 \mathrm{FFU} \mathrm{B2c}$ or $200 \mathrm{FFU}$ DRV. Then the viral load in the mice brain were determined at 3, 5, 6, 7, 8, and 9 d.p.i. The results showed that the genomic RNA of DRV was lower than that of B2c at 3 and 5 d.p.i., but reached the same level as that of B2c at 6 and 7 d.p.i. (Figure 1B). Previous studies have shown that the lab-attenuated RABV infection enhances the $\mathrm{BBB}$ permeability and induces inflammation in the brain $(8,38)$. Thus, the Na-fluorescence (Na-F) uptake of both strains at 7 d.p.i. was measured, and the data showed that Na-F uptake in B2c-infected brain was significantly higher than that of DRV or mock-infected brains (Figure 1C). Consistently, there were more $\mathrm{CD} 45^{+}$lymphocytes in $\mathrm{B} 2 \mathrm{c}$-infected brains than DRV-infected mouse brains (Figures 1D,E). All these results are consistent with the previous findings related to the CNS inflammation and BBB permeability change during comparison of the pathogenicity between wt and lab-attenuated RABV $(6,8,39)$.

\section{B2c Causes Abortive Infection, but DRV Can Persistently Replicate in Primary Astrocytes}

Astrocytes play an important role in the induction of innate immunity in the CNS. To investigate the role of astrocytes in the pathogenesis of RABV, we isolated primary astrocytes and neurons from suckling mice and infected them with DRV or B2c. The growth kinetics of both viruses in astrocytes was assessed by virus titration and immunofluorescence assay (IFA). The viral loads in the cell culture supernatants infected with B2c quickly reached peak at 1 d.p.i., and then gradually decreased until 15 d.p.i. In contrast, the virus titer of DRV was initially relatively low but subsequently increased steadily until the endpoint (Figure 2A). To be noted, the viral titer of DRV in the cell supernatant maintained at a relatively low level than that of B2c in astrocyte at 3 and 5 d.p.i., but $\mathrm{N}$ mRNA transcription levels of DRV was significantly higher than that of B2c at 3 and 5 d.p.i. (Figure 2C). The IFA results showed that DRV persistently replicated in astrocytes and large immunofluorescence foci could be observed at 7 d.p.i., while no obvious immunofluorescence foci could be found in B2c-infected cells (Figure 2D). As a control, virus replication kinetics in primary neurons was also compared. It was found that viral titers of $\mathrm{B} 2 \mathrm{c}$ were always higher than those of wt RABV at the indicated time points (Figure 2B). The IFA results also showed that both DRV and B2c could efficiently replicate in neuron (Figure 2D).

To confirm these observations in vivo, C57BL/6 mice were i.c. infected with $20 \mathrm{FFU}$ B2c or $200 \mathrm{FFU}$ DRV. Infected mice were euthanized when moribund and the brains were harvested for fluorescence IHC analysis. GFAP was a well-known surface marker for astrocytes (44), and the GFAP staining demonstrated DRV could effectively infect astrocytes in the brains (Figure 2E). However, few infected astrocytes could be observed in B2cinfected mouse brains (Figure 2E). In contrast, neuron was intensively infected by both DRV and B2c (Figure 2F). Together, these results show that the $\mathrm{B} 2 \mathrm{c}$ causes abortive infection in astrocytes both in vitro and vivo.

\section{Lab-Attenuated RABV Activates the MAVS Signaling Pathway in Astrocytes}

Previous studies have demonstrated that RABV can be recognized by RIG-I and MDA5, which share a common adaptor MAVS in DCs (23). To assess innate immune responses in astrocytes, cells were infected with DRV or B2c at an MOI of 0.1 and the expression of several proteins involved in the MAVS signaling pathway, namely, RIG-I, p-IRF7, STAT1 and IFIT1 (ISG56), was measured by Western blot. The ubiquitin ligase TRIM25 mediates Lysine 63-linked ubiquitination of RIG-I's $\mathrm{N}$-terminal CARD domains is indispensable to induce type I IFN production and antiviral immunity (45). Thus, the immunoprecipitation of RIG-I was carried out and then resolved by Western blot by using an anti-ubiquitin antibody. As expected, RIG-I was much more robustly ubiquitinated in astrocytes infected with B2c than that in astrocytes infected with DRV. Consistently, the expression levels of RIG-I, p-IRF7, STAT1, and IFIT1 in B2c-infected astrocytes were higher than those in DRV-infected cells (Figure 3A).

Next, we attempted to determine which viral product (RNA or protein) activates MAVS signaling pathway in astrocytes. Primary astrocytes were treated with $\mathrm{CHX}$, a protein synthesis inhibitor, to inhibit viral protein synthesis. It was found that B2c and DRV N transcription levels were similar between CHXtreated and mock-treated astrocytes at $24 \mathrm{~h}$ p.i. (Figure 3B). However, the expression levels of the genes involved in the MAVS signaling pathway, namely, RIG-I, MDA5, MAVS, IRF7, IFN- $\beta$, STAT1, and IFIT1, were significantly upregulated by B2c compared with DRV in both CHX-treated and mock-treated astrocytes, indicating that viral RNA rather than proteins activates IFN pathway depending on MAVS (Figures 3C-I). Taken together, these data demonstrate that the lab-attenuated RABV, but not wt RABV, activates the MAVS signaling pathway by viral RNA, resulting in the production of IFN as well as ISGs in astrocytes.

\section{Lab-Attenuated RABV Produces More dsRNA in Astrocytes}

Retinoic acid-inducible gene I and MDA5 activation is induced mostly by dsRNA, which is produced during viral replication (13). To compare the amount of dsRNA produced by wt and labattenuated RABV, primary astrocytes were infected with DRV or B2c at an MOI of 0.1. At 1 and 3 d.p.i., dsRNA was stained with specific antibody and observed by a confocal fluorescence microscope. The results demonstrate that more dsRNA is synthesized in B2c-infected astrocytes than those in DRV-infected cells (Figure 4A). The dsRNA intensity per cell in B2c-infected cell was significantly higher than that in DRV-infected cells (Figure 4B), considering the similar RABV intensity per cell (Figure 4C). The ratio of dsRNA intensity to RABV intensity in B2c-infected astrocyte was significantly higher than that in DRV-infected cells (Figure 4D). These results suggest that lab-attenuated RABV produces more viral dsRNA than wt RABV during viral replication, 

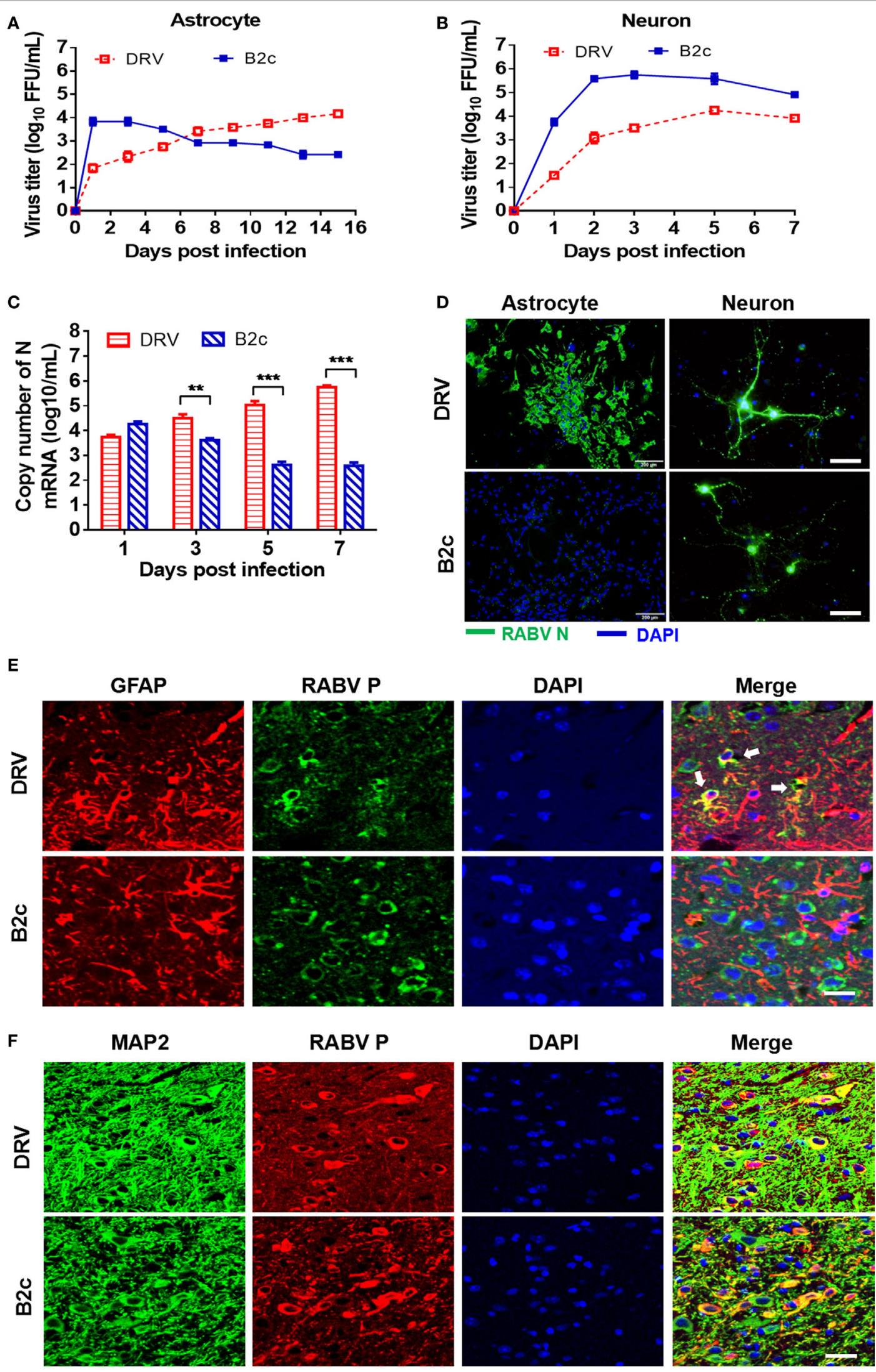

RABV P

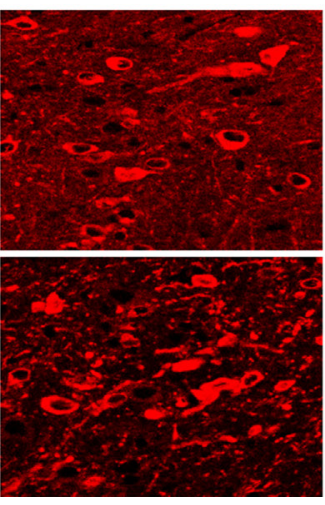

DAPI

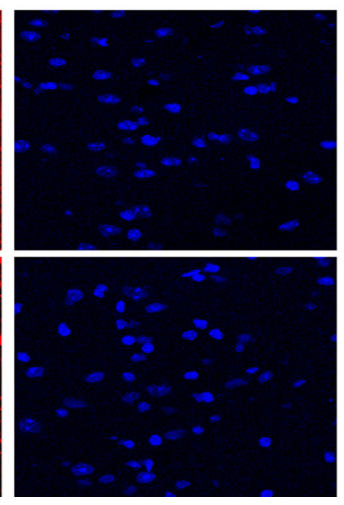

Merge

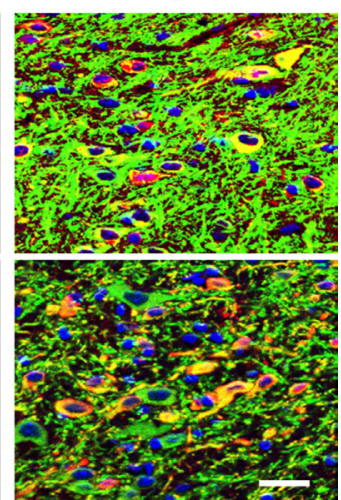


FIGURE 2 | Lab-attenuated rabies virus (RABV) causes abortive infection in astrocytes. Primary astrocytes and neurons were isolated from suckling C57BL/6 mice and then infected with DRV or B2c at an $\mathrm{MOI}$ of 0.01 . (A,B) The cell supernatants from virus infected astrocytes and neurons were collected at indicated time points, and viral titers were determined. (C) RABV N transcription levels in astrocytes were determined by qRT-PCR. (D) At the 7 days postinfection (d.p.i.), astrocytes were fixed with 4\% PFA, stained with an FITC-conjugated anti-RABV N protein antibody (N-FITC) or DAPI and then observed under a fluorescence microscope. The scale bars represent $200 \mu \mathrm{m}$. At $24 \mathrm{~h}$ p.i., neurons were fixed with $4 \%$ paraformaldehyde (PFA) and stained with N-FITC or DAPI. The scale bars represent $50 \mu \mathrm{m}$. Female C57BL/6 mice were infected with DRV (200 FFU) or B2c (20 FFU). (E) At 7 d.p.i., mice were euthanized, perfused with PBS and then fixed with 4\% PFA. The brains were subsequently coated with paraffin, and the brain sections were stained with antibodies against GFAP (red), RABV P protein (green), or DAPI (blue). The white arrows indicate RABV-infected astrocytes. The scale bars represent $50 \mu m$. (F) The same brain sections as (E) were stained with antibodies against MAP2 (green), RABV P protein (red), or DAPI (blue), then visualized under a confocal microscope. The scale bars represent $50 \mu \mathrm{m}$.

which causes the activation of MAVS as well as the downstream signaling.

\section{Lab-Attenuated RABV Can Effectively Replicate in MAVS Knock-Out Astrocytes}

To evaluate the role of MAVS signaling on RABV infections in astrocytes, primary astrocytes isolated from MAVS- - and wt mice were infected with DRV or B2c at an MOI of 0.01 . At 1,3 , and 7 d.p.i., viral titers in the supernatant were measured. Notably, B2c titers were significantly increased in MAVS-/astrocytes compared with wt astrocytes (Figures 5A,B). Moreover, the cell numbers of immunofluorescence plaques in MAVS-/- astrocytes caused by B2c infection were significantly more than those in wt astrocytes (Figures 5E,F). In contrast, MAVS deficiency had no significant influence on DRV replication and spread in astrocytes (Figures $\mathbf{5 A , E}$ ). TBK1 is an IкB kinase downstream of the MAVS signaling pathway and is critical for IRF3 phosphorylation. Treatment with the TBK1 specific inhibitor BX795 significantly increased B2c titers in astrocytes (Figures 5C,D). To verify these observations in vivo, MAVS-/- mice were i.c. infected with DRV or B2c, and virus infection of astrocytes was determined by IFA. We found that both DRV and B2c could efficiently infected MAVS-/- astrocytes (Figures 5G,H). Taken together, these findings suggest that MAVS signaling significantly restricts the replication and spread of lab-attenuated but not wt RABV in astrocytes.

Recent studies have shown that TLR7 may be another innate immune molecule that recognizes RABV (24). Thus, the role of TLR7 in RABV replication was investigated in astrocytes. Astrocytes from TLR7-/- and control mice were isolated and then infected with DRV or B2c at an MOI of 0.01. At different time points p.i., viral titers (Figures 5A,B) and the formation of viral immunofluorescence foci was analyzed (Figures 5 E,F). The results demonstrated that TLR7 deficiency did not significantly affect the replication and spread of either DRV or B2c in astrocytes, suggesting that TLR7 does not play a role in containing RABV replication and spread in astrocytes.

\section{Lab-Attenuated RABV Induces Cytokine Production in Astrocytes}

Astrocytes are one of the major sources of inflammatory cytokines in the CNS post viral infection, and these cytokines play an important role in regulating $\mathrm{BBB}$ permeability. To investigate RABV-induced cytokine production, primary astrocytes from wt and MAVS-/- mice were prepared and infected with $\mathrm{DRV}$ or $\mathrm{B} 2 \mathrm{c}$ at an MOI of 0.01 . At indicated time points, the cell culture supernatants were harvested and analyzed with a cytokine array kit. The results showed that B2c induced significantly higher levels of cytokine expression, namely, TNF- $\alpha$, IL-6, IL-1 $\beta$, IFN- $\gamma$, IL-17, and VEGF expression, than DRV in wt and MAVS-/- astrocytes. The levels of cytokine expression in MAVS-/- astrocytes were significantly lower than those in wt astrocytes, indicating that RABV-induced inflammatory cytokine production in astrocytes is dependent on the MAVS signaling pathway (Figures 6A-F).

The specific pathway through which RABV induces cytokine production was subsequently identified in astrocytes. A previous study demonstrated that RABV induces cytokine production in macrophages mainly through p38, JNK, and NF- $\mathrm{B}$ pathways $(46,47)$. Thus, primary astrocytes were treated with p38, JNK, and NF- $\kappa$ B pathway inhibitors Skepinone-L, JNK IX, and sc75741, respectively, and the concentrations of TNF- $\alpha$ and IL- 6 in the cell supernatant were measured by ELISA. The results showed that Skepinone-L and sc75741 caused greater reductions in TNF- $\alpha$ (Figure 6G) and IL-6 (Figure $6 \mathbf{H}$ ) protein levels than JNK IX in B2c-infected astrocytes. None of these inhibitors significantly altered the expression levels of TNF- $\alpha$ and IL- 6 in DRV-infected astrocytes. These findings suggest that lab-attenuated RABV induces cytokine expression in astrocytes mainly through the $\mathrm{p} 38$ and $\mathrm{NF}-\kappa \mathrm{B}$ pathways and that wt RABV suppresses cytokine production in astrocytes.

\section{Cytokines Produced in Astrocytes Regulate BBB Permeability}

Our previous studies demonstrated that the chemokines/ cytokines induced by $\mathrm{RABV}$ infection are responsible for reducing $\mathrm{TJ}$ protein expression and enhancing $\mathrm{BBB}$ permeability (39). To investigate the effect of these cytokines on BBB permeability, a mouse brain microvascular endothelial cell line b.END3, was cocultured with UV-inactivated supernatants infected with DRV or B2c and collected them at different time points after infection. Treatment with the supernatants from B2c-infected astrocytes for $48 \mathrm{~h}$ (Figure 7A) induced significant increase in Dextran-FITC infiltration from 48 to $96 \mathrm{~h}$ p.i. Notably, treatment with the supernatants from B2c-infected MAVS-/- astrocytes elicited significant increases in DextranFITC permeability at $48 \mathrm{~h}$ p.i. (Figure 7B). No significant increases in Dextran-FITC permeability were observed after treatment with the supernatants from wt or MAVS-/- astrocytes infected with DRV.

To gain an insight into the mechanisms by which labattenuated $\mathrm{RABV}$ enhances $\mathrm{BBB}$ permeability, the expression 

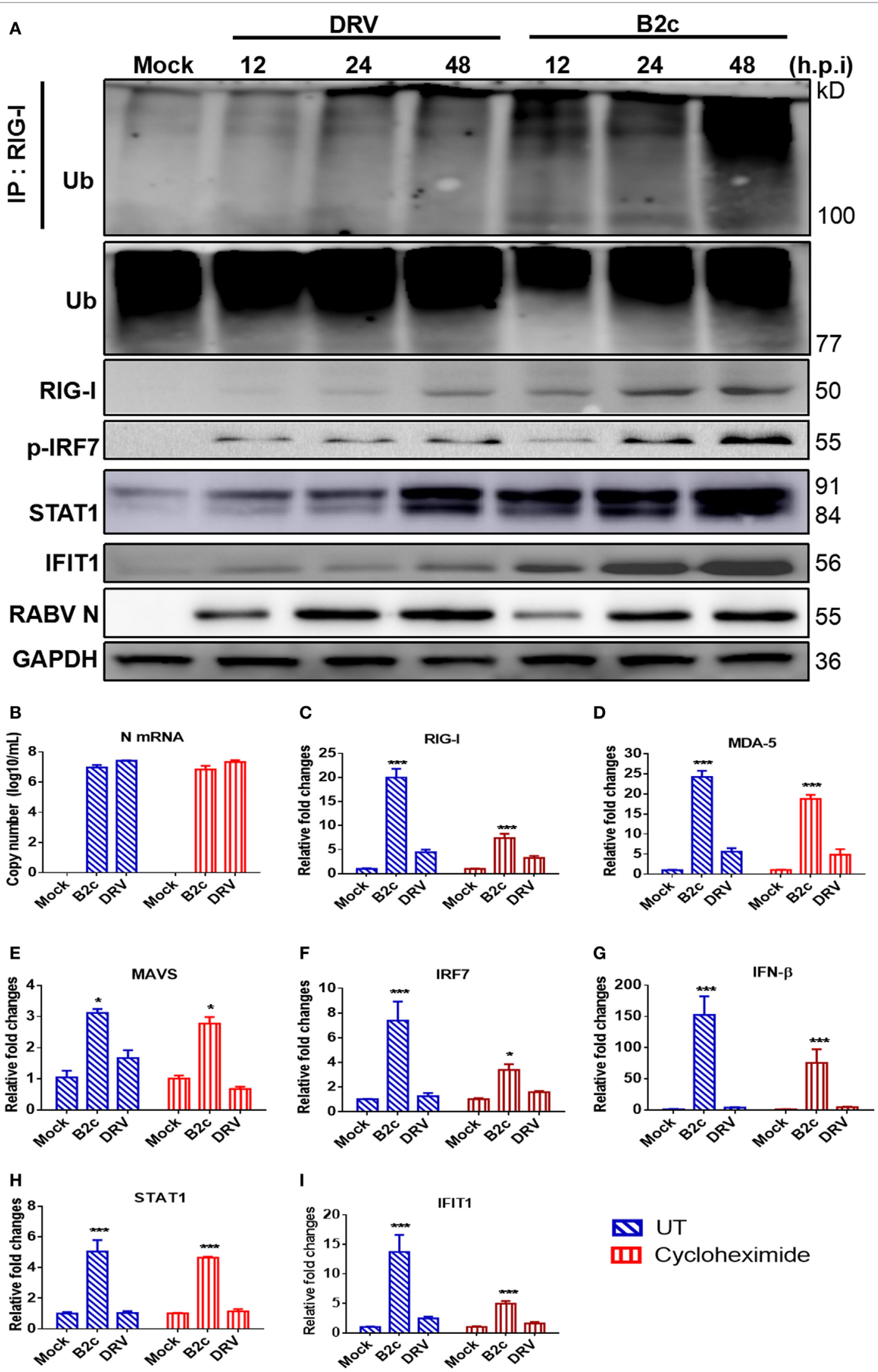

\$ UT

In Cycloheximide 
FIGURE 3 | Lab-attenuated rabies virus (RABV) causes the activation of mitochondrial antiviral-signaling protein (MAVS) signaling pathway in astrocytes. Primary astrocytes were infected with DRV or B2c at an $\mathrm{MOI}$ of 0.1 . (A) At the indicated time points, the cells were lysed with RIPA buffer, the activation of retinoic acid-inducible gene I (RIG-I) was characterized by immunoprecipitation with an anti-RIG-I antibody and then immunostaining with an anti-UBC antibody. The protein expression level of the key molecules in MAVS signaling pathway was assessed by Western blot using antibodies against RIG-I, phosho-IRF7, STAT1, IFIT1, RABV N, and GAPDH. (B) RABV N mRNA transcription levels were measured by qRT-PCR after the cells were treated with cycloheximide or mock-controls. (C-I) The fold changes in RIG-I, melanoma differentiation-associated protein 5, MAVS, IRF7, interferon- $\beta$, STAT1, and IFIT1 transcription levels were measured by qRT-PCR. The qRT-PCR results were calculated by the $\Delta \Delta \mathrm{C}_{\mathrm{t}}$ method and expressed as $2^{-\Delta \Delta \mathrm{Ct}}$.

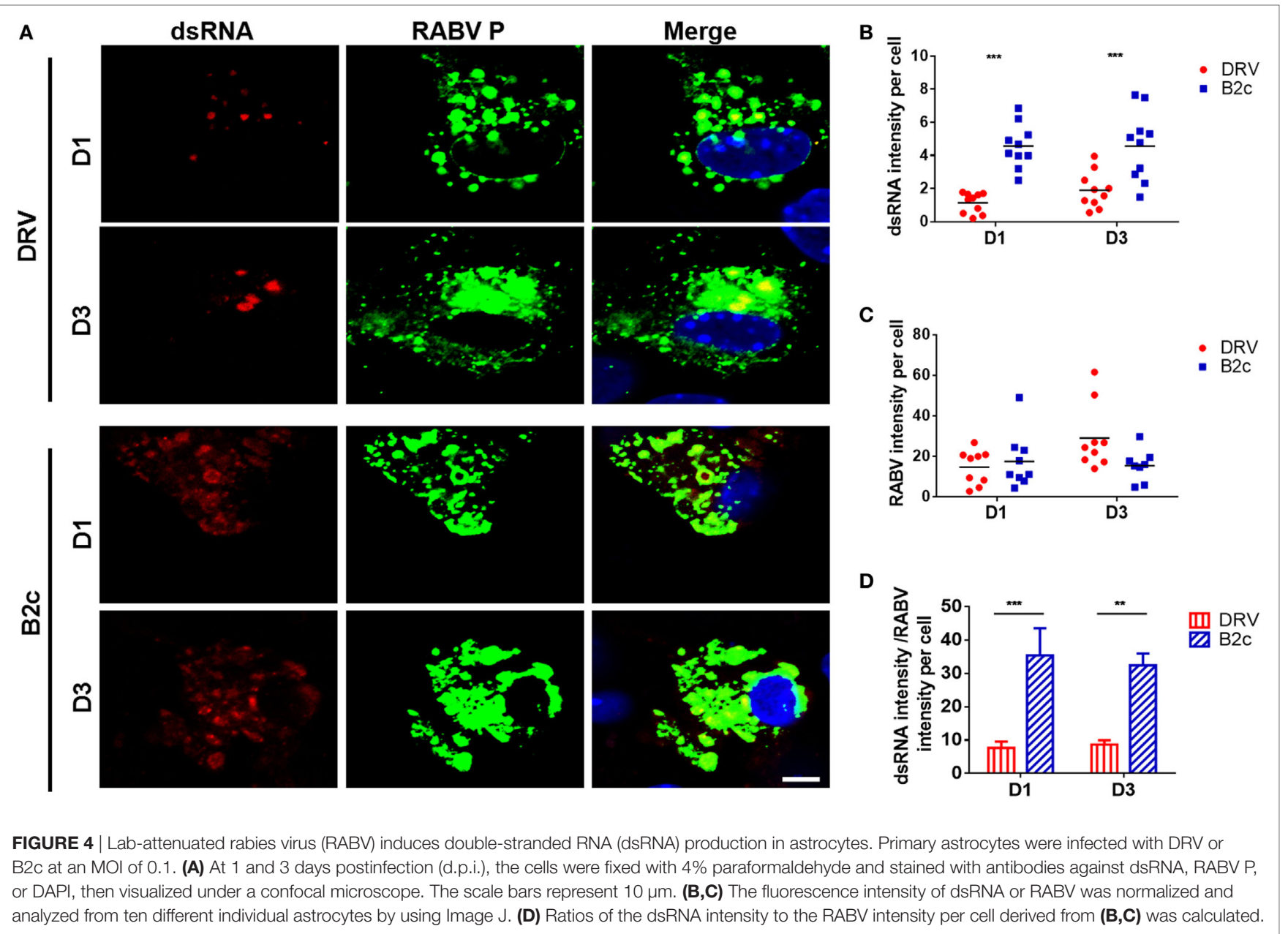

levels of the indicated TJ proteins (Claudin-5, Occludin, and $\mathrm{ZO}-1$ ) were assessed in astrocytes by Western blot. It was found that the expression levels of Claudin-5 and Occludin were unchanged, while ZO-1 was significantly reduced after the cells were treated with the supernatants from B2c-infected wt astrocyte (Figure 7C). However, this reduction was attenuated in cells treated with the supernatants from MAVS-/- astrocytes infected with B2c. No significant changes in ZO-1 expression were observed in b.END3 cells treated with supernatants from wt or MAVS-/- astrocyte infected with DRV (Figure 7D).

Similarly, IFA results showed that ZO-1 expression was significantly decreased in b.END3 cells cocultured with the supernatants from B2c-infected astrocytes collected at $48 \mathrm{~h}$ p.i. (Figure 7E). ZO-1 fluorescence intensity was only slightly decreased in b.END3 cells cocultured with the supernatants from B2c-infected MAVS-/- astrocytes collected at $48 \mathrm{~h}$ p.i., indicating that cell-to-cell contact was not significantly decreased in these cells. Moreover, no ZO-1 degradation was observed in b.END3 cells cocultured with the supernatants from wt or MAVS-/- astrocytes infected with DRV. To be noted, no significant changes in Claudin-5 and Occludin expression were detected in either wt or MAVS-/- astrocytes upon RABV infection (Figures 7C,E). Together, these results illustrate that lab-attenuated, but not wt, RABV upregulates the production of inflammatory cytokines in astrocytes, resulting in ZO-1 degradation in BMECs and subsequent increases in BBB permeability. Furthermore, these results indicate that cytokine production is dependent on MAVS signaling pathway. 

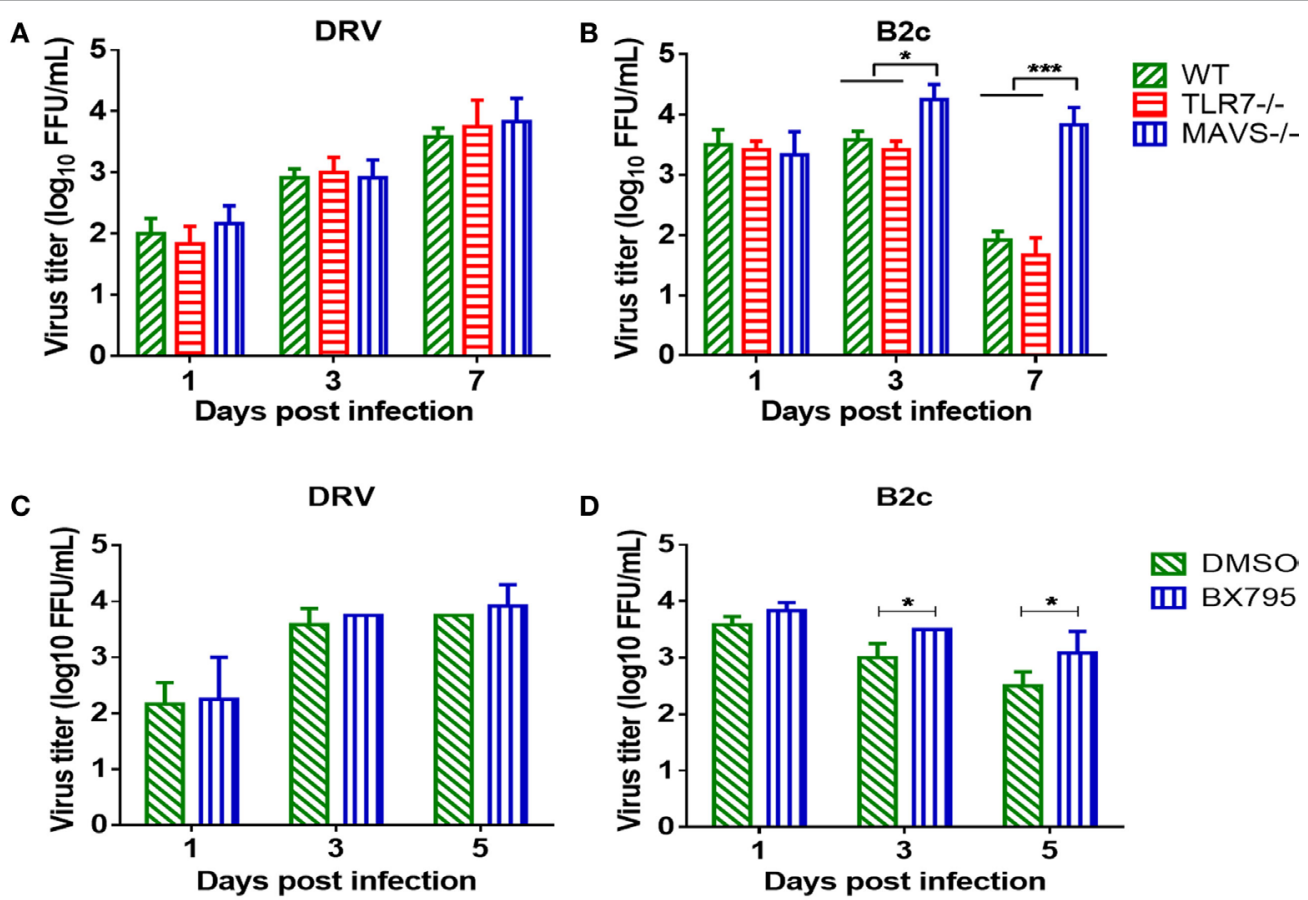

D

B2c
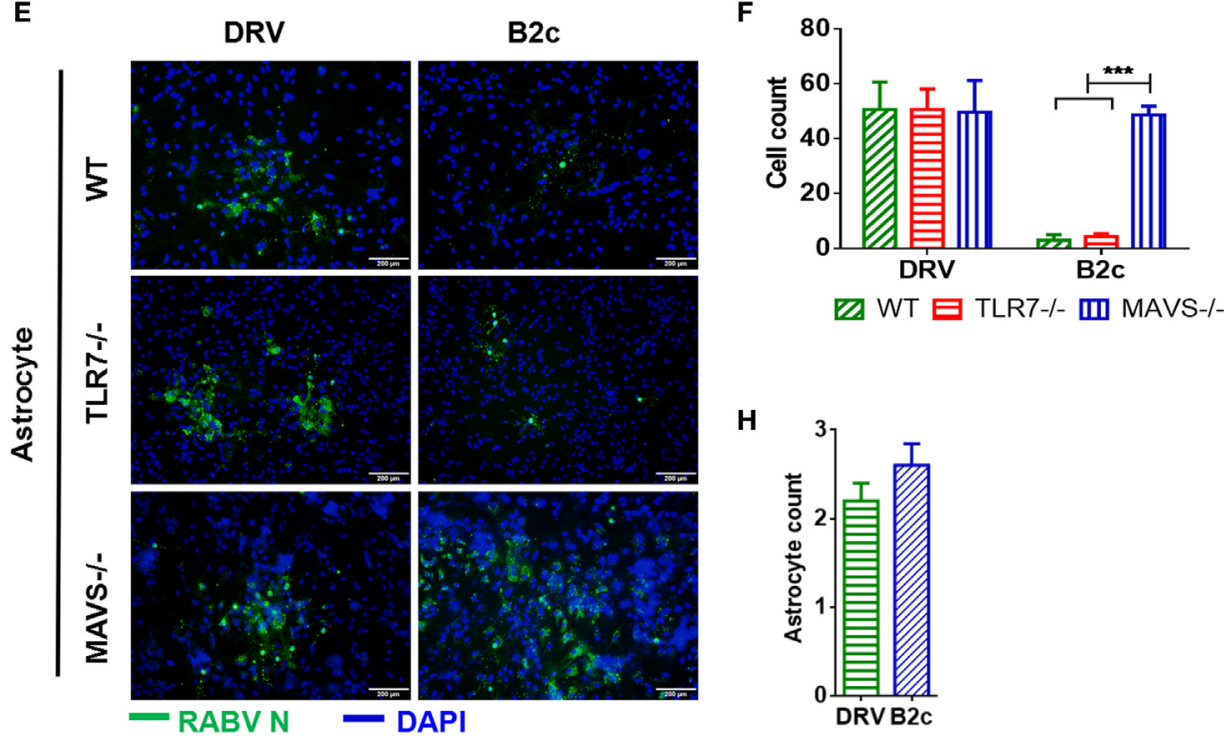

G

GFAP

RABV P

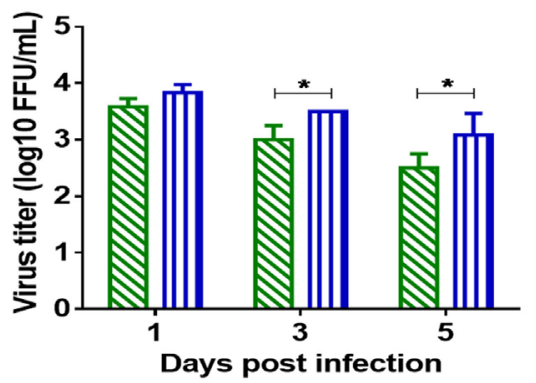

DMSO

血 BX795

H
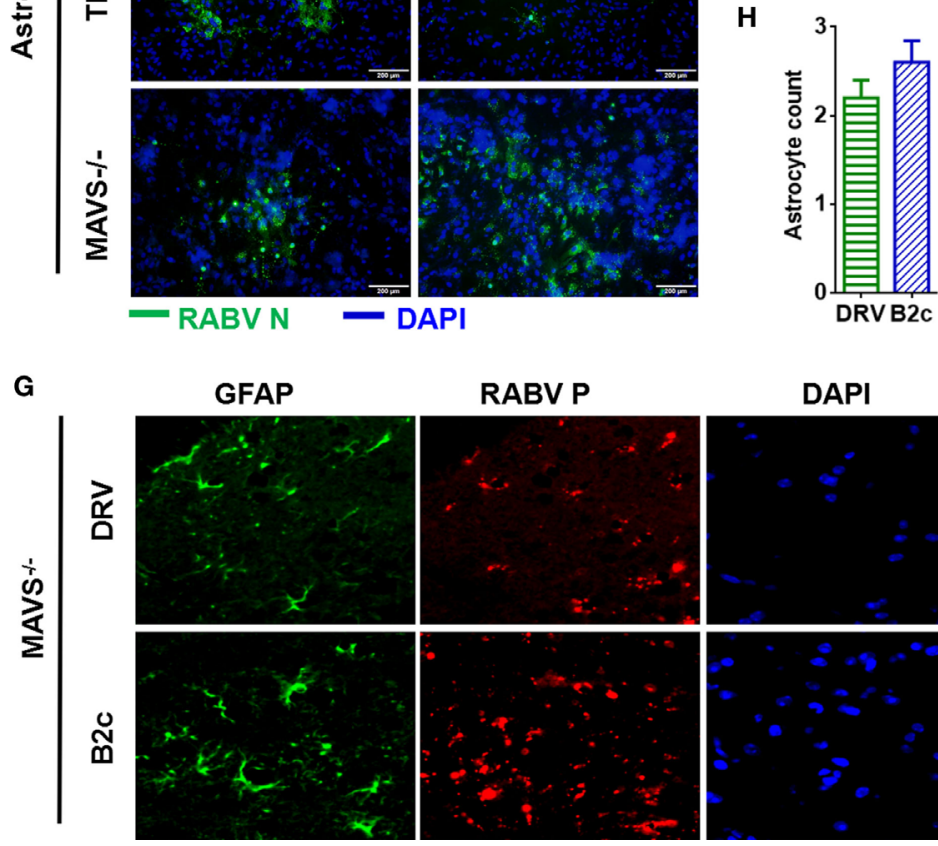

DAPI

Merge
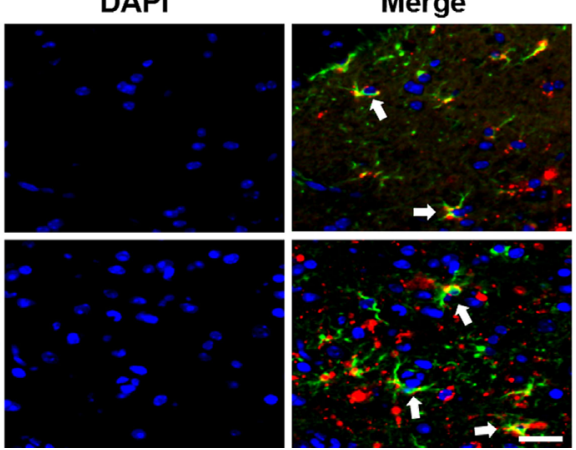
FIGURE 5 | Lab-attenuated rabies virus (RABV) infects astrocytes lacking mitochondrial antiviral-signaling protein (MAVS) in the same manner as wt RABV. (A,B) Wild-type (wt), MAVS-/-, or TLR7-/- astrocytes were infected with DRV or B2c at an MOI of 0.01. At the indicated time points, the viral titers in the cell culture supernatant were determined by virus titration. (C,D) Primary astrocytes were treated with BX795 at a dose of $1 \mu \mathrm{M}$ or DMSO, and then infected with DRV or B2c at an $\mathrm{MOI}$ of 0.01 , at the indicated time points, the viral titers in the cell culture supernatant were determined by virus titration. (E,F) Wt, TLR7-/- or MAVS-/- astrocytes were infected with DRV or B2C at an MOI of 0.01, fixed with paraformaldehyde and then stained with anti-RABV N antibodies or DAPI, and the viral infected cells were calculated from five different areas. The scale bars represent $200 \mu \mathrm{m}$. (G,H) At 7 days postinfection, the cerebral cortex sections from MAVS-/- mice were subsequently stained with antibodies against GAFP (red), RABV P (green), or DAPI (blue), and then visualized under a confocal microscope, and the viral infected astrocytes were calculated from five different areas. The white arrow indicates RABV-infected astrocytes. The scale bars represent $50 \mu \mathrm{m}$. The infected cells were quantified by ImageJ software and statistical analysis was applied.

A

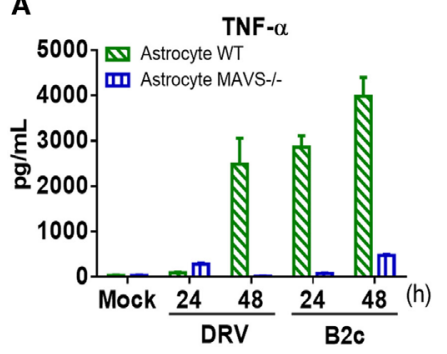

D

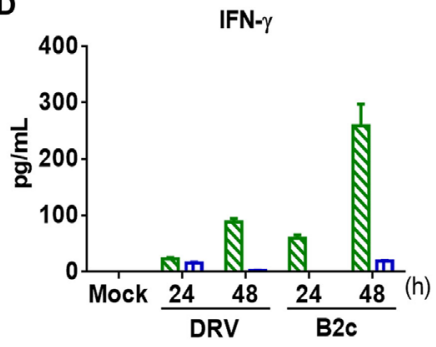

G

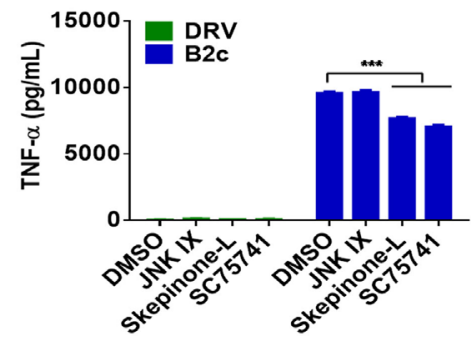

B

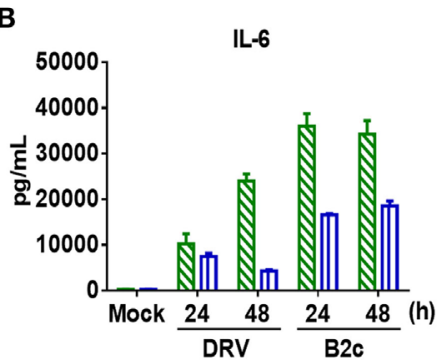

E

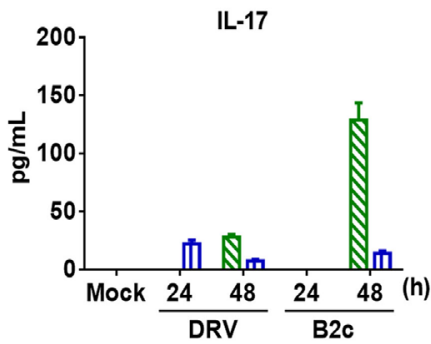

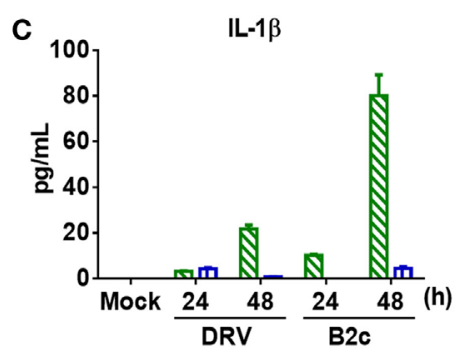

F

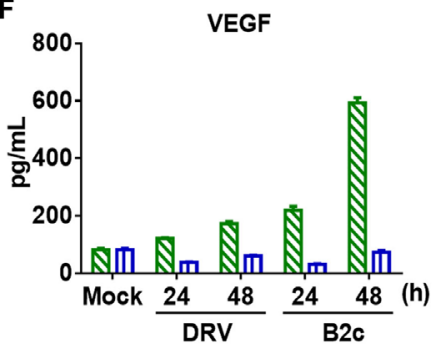

H

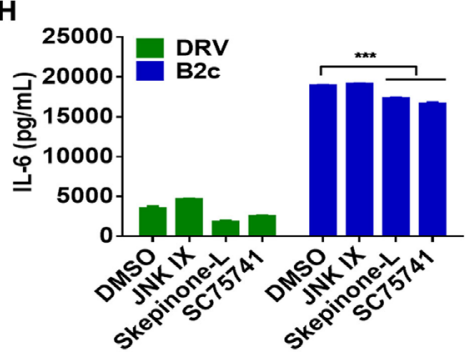

FIGURE 6 | Cytokine expression in astrocytes infected by lab-attenuated rabies virus (RABV) is dependent on the mitochondrial antiviral-signaling protein (MAVS)-p38/NF-кB pathway. (A-F) Wild-type (wt) or MAVS-/- astrocytes were infected with DRV or B2c at an MOI of 1. At the indicated time points, the cell supernatants were collected, and TNF- $\alpha, \mathrm{IL}-6, \mathrm{IL}-1 \beta$, interferon- $\gamma, \mathrm{IL}-17$, and vascular endothelial growth factor levels were determined by protein assay. (G, $\mathbf{H})$ Wt astrocytes were treated with JNK inhibitor IX (JNK inhibitor), Skepinone-L (p38 inhibitor), sc75741 (NF-kB inhibitor), or DMSO (mock), after being infected with DRV and $\mathrm{B} 2 \mathrm{c}$ at an $\mathrm{MOI}$ of 1 . At $48 \mathrm{~h}$ p.i., the concentrations of TNF- $\alpha$ and IL-6 in the cell supernatant were measured with ELISA kits.

\section{DISCUSSION}

Astrocytes play critical roles in host defense during viral infections of the CNS (7). PRR activation in astrocytes results in the expression of many immune mediators, including type I IFNs and inflammatory cytokines $(6,7)$. During infections caused by pathogens for which glia are not susceptible targets, activation of the innate immune system caused by pathogen recognition in astrocytes may promote antiviral immune responses in susceptible neurons, as well as CNS leukocyte trafficking $(3,5,7)$. In an early report, primary murine, feline, and human astrocytes were infected with wt (SRV) and lab-attenuated RABV (ERA) (34), after which viral loads and replication were assessed by infectivity assay and immunofluorescence. The results showed that astrocytes can be infected by RABV, suggesting that astrocytes may play a role in viral spread and persistence and/or neuronal dysfunction (34). However, in that study, viral loads and replication were evaluated only at the early time point after infection (3 d.p.i.). In this present study, the growth of DRV with that of B2c, which were used as a pair of wt and lab-attenuated RABV in previous studies (38-40), was compared in a long-term experiment. Surprisingly, we found that lab-attenuated RABV, but not 

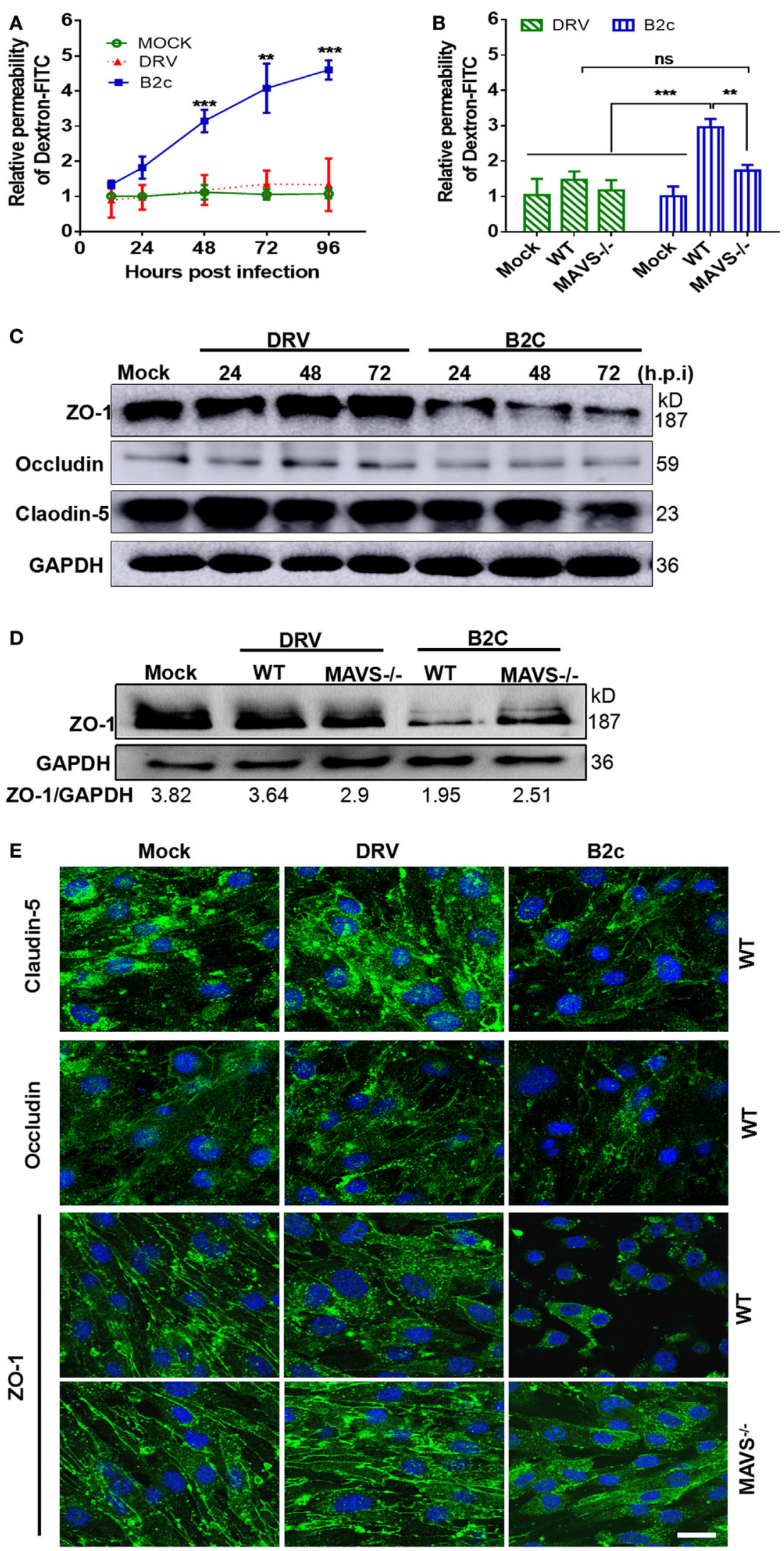
FIGURE 7 | Inflammatory cytokines produced by astrocytes play a critical role in regulating blood-brain barrier permeability in rabies virus infection. Wild-type or mitochondrial antiviral-signaling protein (MAVS-/-) astrocytes were infected with DRV or B2c at an MOI of 1 or mock infected. (A,B) At the indicated time points, the cell supernatants were collected and UV-inactivated, then they were cocultured with b.END3 cell for $48 \mathrm{~h}$. The cells were treated with Dextran-FITC at a dose of $0.1 \mu \mathrm{g} / \mathrm{mL}$ for $30 \mathrm{~min}$, and then the Dextran-FITC fluorescence values in the media from the upper and lower chambers were determined by a fluorimeter. (C,D) The b.END3 cells were lysed with RIPA buffer, and the protein expression levels were determined by Western blot using antibodies against Zoluna occludens-1 (ZO-1), Claudin-5, Occludin, or GAPDH. (E) The b.END3 cells were fixed with PFA, stained with antibodies against Claudin-5, Occludin, ZO-1, or DAPI, and then observed under an immunofluorescence microscope. The scale bars represent $50 \mu \mathrm{m}$.

wt RABV, caused abortive replication in astrocytes, a feature that may be associated with the ability of the virus to evade the innate immune response.

Productive infection of the astrocyte is critical for neurotropic pathogens to induce encephalitis. Astrocytes sensed viral entry into the CNS and mounted a type I IFN response, which rapidly restricts the virus after neuronal transport into the CNS. Previous studies demonstrated that TLR3-/- astrocytes were more permissive to HSV infection and caused severe symptom of encephalitis and tissue damage, which was due to impaired type I IFN production in the absence of TLR3 (30). A recently work found that abortively infected astrocytes are the major producers of IFN- $\beta$ after infection of the brain with diverse neurotropic viruses, including TMEV, RABV, and vesicular stomatitis virus (VSV) (35). Consistent with these studies, we also found that the abortive infection of lab-attenuated RABV in astrocytes was related to its ability to activate IFN signaling pathway. Basal ISG expression levels are an important determinant of susceptibility to viral infection. We have found that astrocytes have higher basal expression levels of the mRNAs encoding ISG proteins, such MDA5 and STAT1, and other molecules crucial for recognizing viral invasion and creating an more antiviral environment than neurons (9), which may explain why lab-attenuated RABV activates the innate immune response to a degree sufficient to restrict viral replication in astrocytes but not in neurons.

Double-stranded RNA is a viral product that plays an essential role in inducing innate immunity, which leads to the production of type I IFNs and the activation of hundreds of ISGs. Early biochemical studies of viral replication suggested that most viruses produce dsRNAs (13). However, in 2006, Weber et al. reported that dsRNA could be detected by IFA in cells infected with positive-stranded RNA viruses, but not with negative-stranded RNA viruses (48). This notion was challenged by two other studies on dsRNA production in cells infected with negative-stranded RNA viruses $(13,48)$. Moreover, a recent study demonstrated the dsRNA formation in cells infected with several negative-stranded RNA viruses, such as VSV, measles virus $(\mathrm{MeV})$, and influenza $\mathrm{A}$ virus, although the intensity of the staining of dsRNA tended to be weaker in cells infected with negative-stranded RNA viruses when compared with those infected with positive-stranded RNA viruses (13). Consistent with this finding, the production of dsRNA was detected in both lab-attenuated and wt RABV-infected astrocytes, although lab-attenuated RABV produced significantly more dsRNA in the cytoplasm than wt RABV.

We attempted to investigate why lab-attenuated RABV produces more dsRNA than wt RABV. Pfaller et al. found that dsRNA expression was much lower in cells infected with wt $\mathrm{MeV}$ than in cells infected with a mutant $\mathrm{MeV}$ whose $\mathrm{C}$ protein was knocked out which is known to control genome replication and transcription (49). Similarly, Takeuchi et al. observed dsRNA formation in cells infected with Sendai virus (50) with C protein knocked out but not in cells infected with wt $\mathrm{SeV}$, indicating that the $\mathrm{C}$ protein limits or masks dsRNA production (51). Both $\mathrm{MeV}$ and $\mathrm{SeV}$ are within the family Paramyxoviridae, thus it is possible that their $\mathrm{C}$ protein possess the similar function to subvert the production of dsRNA. Ebola virus protein VP35 adopts a unique strategy to mask key cellular recognition sites on dsRNA (52). A recently study showed that the coronavirus endonuclease (EndoU) activity is the key to prevent early induction of dsRNA. Replication of EndoU-deficient coronaviruses is greatly attenuated in vivo and severely restricted in primary cells even during the early phase of virus infection (53). In this present study, by exchanging viral genes between wt and lab-attenuated RABV, we found that single $\mathrm{N}, \mathrm{P}$, and $\mathrm{G}$ of wt RABV could not suppress dsRNA formation (data not shown). However, multiple viral proteins of RABV may work together to limit the production of dsRNA. Further studies are needed to elucidate the mechanism through which wt RABV restricts dsRNA formation and thus evades recognition by the innate immune system in infected cells.

The BBB, which is composed of specialized BMECs joined by TJs and ensheathed by astrocytes and pericytes, plays an important role in protecting the CNS. Our previous studies have shown that RABV does not infect BMECs, nor does it modulate TJ protein expression in BMECs (39). However, brain extracts prepared from mice infected with lab-attenuated RABV but not wt $\mathrm{RABV}$ reduced TJ protein expression in BMECs, indicating that the above enhancements of $\mathrm{BBB}$ permeability and reductions in $\mathrm{TJ}$ protein expression are not caused by RABV infection. Rather, they are caused by virus-induced inflammatory chemokines/ cytokines. The innate immune mechanisms that regulate $\mathrm{BBB}$ function in the setting of infectious diseases have been appreciated only recently. Multiple inflammatory cytokines, including TNF- $\alpha$, IL-6, IL- $1 \beta$, IFN- $\gamma$, IL-17, and VEGF, disrupt BBB and TJ integrity in BMECs $(46,50,54-56)$, and inflammatory cytokine signaling at the $\mathrm{BBB}$ during infection facilitates leukocyte trafficking into the CNS, which is essential for the clearance of many pathogens $(39,41)$. Our present study demonstrates that lab-attenuated RABV induces production of several inflammatory cytokines in astrocytes, especially TNF- $\alpha$, IL-6, IL- $1 \beta$, IFN- $\gamma$, IL-17, and VEGF, which cause disruption of the BBB and TJ integrity. Our findings suggest that astrocytes play a critical role in regulating $\mathrm{BBB}$ permeability as a major source of cytokines during viral infection. Furthermore, we found that the production of inflammatory cytokines in astrocytes by lab-attenuated RABV was dependent on MAVS signaling pathway, underscoring the critical role of MAVS signaling in defensing against RABV infection in CNS. 


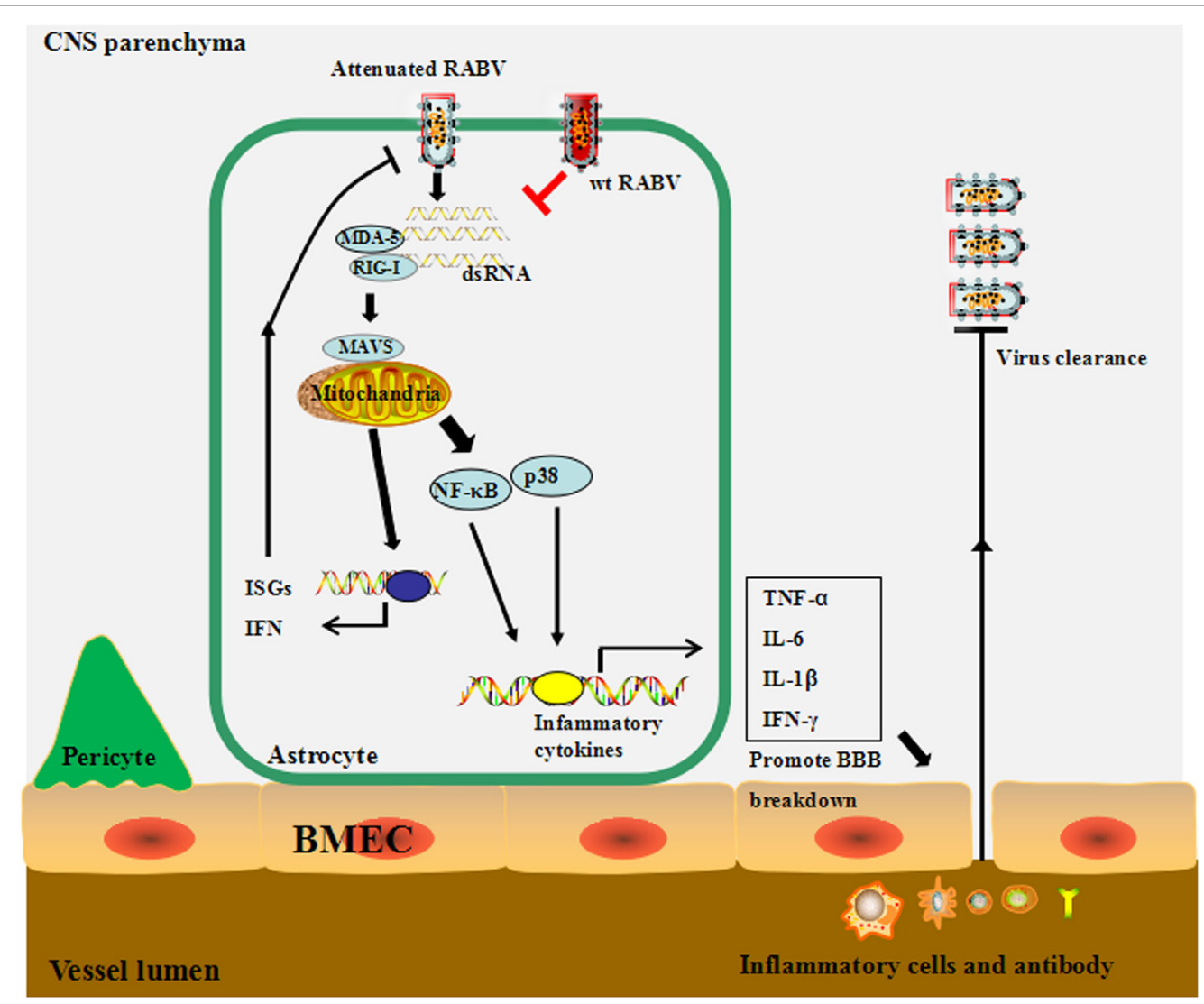

FIGURE 8 | The proposed model for the mechanism through which wild-type (wt) and lab-attenuated rabies virus (RABV) infect astrocytes. During infection, lab-attenuated RABV produces double-stranded RNA (dsRNA), which is recognized by retinoic acid-inducible gene I (RIG-I)/melanoma differentiation-associated protein 5 (MDA5). Activation of mitochondrial antiviral-signaling protein (MAVS), the common adaptor protein for RIG-I and MDA5, leads to enhanced production of interferon, as well as some IFN-stimulated genes, which limit RABV replication and spread. MAVS activation stimulates the p38 and NF- $\mathrm{BB}$ signaling pathways and induces cytokine production in astrocytes. Inflammatory cytokines promote blood-brain barrier (BBB) permeability, enabling peripheral inflammatory cells and antibodies to infiltrate into the central nervous system, thereby facilitating RABV clearance. In contrast, wt RABV prevents activation of the MAVS signaling pathway by restricting dsRNA production.

\section{CONCLUSION}

Based on the results, we propose the following model: labattenuated RABV produces dsRNA recognized by RIG-I, MDA5, or both, resulting in the activation of the MAVS signaling pathway in astrocytes. IFN expression induces the transcription of hundreds of ISGs to inhibit RABV replication in astrocytes and causes the abortive infection by lab-attenuated RABV. The inflammatory cytokines induced by lab-attenuated RABV enhance BBB permeability, enabling immune cells and antibodies to infiltrate the CNS and facilitate RABV clearance. Conversely, wt RABV restricts dsRNA production and then evades recognition by the innate immune system, resulting in persistent viral replication in astrocytes (Figure 8).

\section{ETHICS STATEMENT}

All experiments involving mice were performed in accordance with the recommendations in the Guide for the Care and Use of Laboratory Animals of the Ministry of Science and Technology of China and were approved by the Scientific Ethics Committee of
Huazhong Agricultural University (permit number HZAUMO2015-019).

\section{AUTHOR CONTRIBUTIONS}

Conceived and designed the experiments: BT and LZ. Performed the experiments: MZ, YY, LY, ZL, DT, and KW. Analyzed the data: BT, MC, HC, ZF, and LZ. Wrote the article: BT, MZ, ZF, and LZ.

\section{ACKNOWLEDGMENTS}

The authors wish to thank the staff members of the animal facility at Huazhong Agricultural University for caring for the mice.

\section{FUNDING}

This work was partially supported by the National Natural Science Foundation of China (31720103917 and 31330078 to ZF and 31372419 and 31522057 to LZ); the National Program on key Research Project of China (2016YFD0500400 to LZ). 


\section{REFERENCES}

1. Fooks AR, Banyard AC, Horton DL, Johnson N, McElhinney LM, Jackson AC. Current status of rabies and prospects for elimination. Lancet (2014) 384(9951):1389-99. doi:10.1016/S0140-6736(13)62707-5

2. Schnell MJ, McGettigan JP, Wirblich C, Papaneri A. The cell biology of rabies virus: using stealth to reach the brain. Nat Rev Microbiol (2010) 8(1):51-61. doi:10.1038/nrmicro2260

3. Rupprecht CE. Rhabdoviruses: rabies virus. In: Baron S, editor. The Medical Microbiology. 4th ed. Galveston, TX: University of Texas Medical Branch at Galveston Press (1996). p. 780-90.

4. Suja MS, Mahadevan A, Madhusudana SN, Shankar SK. Role of apoptosis in rabies viral encephalitis: a comparative study in mice, canine, and human brain with a review of literature. Patholog Res Int (2011) 2011:374286. doi:10.4061/2011/374286

5. Yan X, Prosniak M, Curtis MT, Weiss ML, Faber M, Dietzschold B, et al. Silver-haired bat rabies virus variant does not induce apoptosis in the brain of experimentally infected mice. J Neurovirol (2001) 7(6):518-27. doi:10.1080/135502801753248105

6. Wang ZW, Sarmento L, Wang Y, Li XQ, Dhingra V, Tseggai T, et al. Attenuated rabies virus activates, while pathogenic rabies virus evades, the host innate immune responses in the central nervous system. J Virol (2005) 79(19):12554-65. doi:10.1128/JVI.79.19.12554-12565.2005

7. Sarmento L, Tseggai T, Dhingra V, Fu ZF. Rabies virus-induced apoptosis involves caspase-dependent and caspase-independent pathways. Virus Res (2006) 121(2):144-51. doi:10.1016/j.virusres.2006.05.002

8. Roy A, Phares TW, Koprowski H, Hooper DC. Failure to open the bloodbrain barrier and deliver immune effectors to central nervous system tissues leads to the lethal outcome of silver-haired bat rabies virus infection. J Virol (2007) 81(3):1110-8. doi:10.1128/JVI.01964-06

9. Zhao L, Toriumi H, Kuang Y, Chen H, Fu ZF. The roles of chemokines in rabies virus infection: overexpression may not always be beneficial. J Virol (2009) 83(22):11808-18. doi:10.1128/JVI.01346-09

10. Jackson AC, Randle E, Lawrance G, Rossiter JP. Neuronal apoptosis does not play an important role in human rabies encephalitis. J Neurovirol (2008) 14(5):368-75. doi:10.1080/13550280802216502

11. Takeda K, Akira S. Toll-like receptors in innate immunity. Int Immunol (2005) 17(1):1-14. doi:10.1093/intimm/dxh186

12. Kaisho T, Akira S. Toll-like receptor function and signaling. J Allergy Clin Immunol (2006) 117(5):979-87. doi:10.1016/j.jaci.2006.02.023

13. Kyung-No SZ, Liang Z, Lipton HL. Double-strand RNA is detected by immunofluorescence analysis in RNA and DNA virus infections including those by negative-strand RNA viruses. J Virol (2015) 89(18):9383-92. doi:10.1128/ JVI.01299-15

14. Johnson AC, Li X, Pearlman E. MyD88 functions as a negative regulator of TLR3/TRIF-induced corneal inflammation by inhibiting activation of c-Jun N-terminal kinase. J Biol Chem (2008) 283(7):3988-96. doi:10.1074/jbc. M707264200

15. Saito T, Hirai R, Loo YM, Owen D, Johnson CL, Sinha SC, et al. Regulation of innate antiviral defenses through a shared repressor domain in RIG-I and LGP2. Proc Natl Acad Sci U S A (2007) 104(2):582-7. doi:10.1073/pnas.0606699104

16. Ikeda F, Hecker CM, Rozenknop A, Nordmeier RD, Rogov V, Hofmann K, et al. Involvement of the ubiquitin-like domain of TBK1/IKK-i kinases in regulation of IFN-inducible genes. EMBO J (2007) 26(14):3451-62. doi:10.1038/ sj.emboj.7601773

17. Schlee M, Roth A, Hornung V, Hagmann CA, Wimmenauer V, Barchet W, et al. Recognition of 5' triphosphate by RIG-I helicase requires short blunt double-stranded RNA as contained in panhandle of negative-strand virus. Immunity (2009) 31(1):25-34. doi:10.1016/j.immuni.2009.05.008

18. Li X, Lu C, Stewart M, Xu H, Strong RK, Igumenova T, et al. Structural basis of double-stranded RNA recognition by the RIG-I like receptor MDA5. Arch Biochem Biophys (2009) 488(1):23-33. doi:10.1016/j.abb.2009.06.008

19. Hou F, Sun L, Zheng H, Skaug B, Jiang QX, Chen ZJ. MAVS forms functional prion-like aggregates to activate and propagate antiviral innate immune response. Cell (2011) 146(3):448-61. doi:10.1016/j.cell.2011.06.041

20. Paz S, Sun Q, Nakhaei P, Romieu-Mourez R, Goubau D, Julkunen I, et al. Induction of IRF-3 and IRF-7 phosphorylation following activation of the RIG-I pathway. Cell Mol Biol (Noisy-le-grand) (2006) 52(1):17-28. doi:10.1170/T694
21. Prehaud C, Megret F, Lafage M, Lafon M. Virus infection switches TLR-3positive human neurons to become strong producers of beta interferon. J Virol (2005) 79(20):12893-904. doi:10.1128/JVI.79.20.12893-12904.2005

22. Menager P, Roux P, Megret F, Bourgeois JP, Le Sourd AM, Danckaert A, et al. Toll-like receptor 3 (TLR3) plays a major role in the formation of rabies virus Negri Bodies. PLoS Pathog (2009) 5(2):e1000315. doi:10.1371/journal. ppat. 1000315

23. Faul EJ, Wanjalla CN, Suthar MS, Gale M, Wirblich C, Schnell MJ. Rabies virus infection induces type I interferon production in an IPS-1 dependent manner while dendritic cell activation relies on IFNAR signaling. PLoS Pathog (2010) 6(7):e1001016. doi:10.1371/journal.ppat.1001016

24. Li J, Faber M, Dietzschold B, Hooper DC. The role of toll-like receptors in the induction of immune responses during rabies virus infection. Adv Virus Res (2011) 79:115-26. doi:10.1016/B978-0-12-387040-7.00007-X

25. Pardridge WM. Blood-brain barrier biology and methodology. J Neurovirol (1999) 5(6):556-69. doi:10.3109/13550289909021285

26. Jensen CJ, Massie A, De Keyser J. Immune players in the CNS: the astrocyte. J Neuroimmune Pharmacol (2013) 8(4):824-39. doi:10.1007/s11481013-9480-6

27. Borrow P, Nash AA. Susceptibility to Theiler's virus-induced demyelinating disease correlates with astrocyte class II induction and antigen presentation. Immunology (1992) 76(1):133-9.

28. Caccuri RL, Iacono RF, Weissenbacher MC, Avila MM, Berria MI. Longlasting astrocyte reaction to persistent Junin virus infection of rat cortical neurons. JNeural Transm (Vienna) (2003) 110(8):847-57. doi:10.1007/ s00702-003-0010-1

29. Gorry PR, Ong C, Thorpe J, Bannwarth S, Thompson KA, Gatignol A, et al. Astrocyte infection by HIV-1: mechanisms of restricted virus replication, and role in the pathogenesis of HIV-1-associated dementia. Curr HIV Res (2003) 1(4):463-73. doi:10.2174/1570162033485122

30. Reinert LS, Harder L, Holm CK, Iversen MB, Horan KA, Dagnaes-Hansen F, et al. TLR3 deficiency renders astrocytes permissive to herpes simplex virus infection and facilitates establishment of CNS infection in mice. J Clin Invest (2012) 122(4):1368-76. doi:10.1172/JCI60893

31. Gardner J, Borgmann K, Deshpande MS, Dhar A, Wu L, Persidsky R, et al. Potential mechanisms for astrocyte-TIMP-1 downregulation in chronic inflammatory diseases. J Neurosci Res (2006) 83(7):1281-92. doi:10.1002/ jnr.20823

32. Spindler KR, Hsu TH. Viral disruption of the blood-brain barrier. Trends Microbiol (2012) 20(6):282-90. doi:10.1016/j.tim.2012.03.009

33. Pozner RG, Collado S, Jaquenod de Giusti C, Ure AE, Biedma ME, Romanowski V, et al. Astrocyte response to Junin virus infection. Neurosci Lett (2008) 445(1):31-5. doi:10.1016/j.neulet.2008.08.059

34. Ray NB, Power C, Lynch WP, Ewalt LC, Lodmell DL. Rabies viruses infect primary cultures of murine, feline, and human microglia and astrocytes. Arch Virol (1997) 142(5):1011-9. doi:10.1007/s007050050136

35. Pfefferkorn C, Kallfass C, Lienenklaus S, Spanier J, Kalinke U, Rieder M, et al. Abortively infected astrocytes appear to represent the main source of interferon beta in the virus-infected brain. J Virol (2015) 90(4):2031-8. doi:10.1128/JVI.02979-15

36. Yu F, Zhang G, Xiao S, Fang L, Xu G, Yan J, et al. Complete genome sequence of a street rabies virus isolated from a rabid dog in China. J Virol (2012) 86(19):10890-1. doi:10.1128/JVI.01775-12

37. Zhang R, Liu C, Cao Y, Jamal M, Chen X, Zheng J, et al. Rabies viruses leader RNA interacts with host Hsc70 and inhibits virus replication. Oncotarget (2017) 8(27):43822-37. doi:10.18632/oncotarget.16517

38. Kuang Y, Lackay SN, Zhao L, Fu ZF. Role of chemokines in the enhancement of $\mathrm{BBB}$ permeability and inflammatory infiltration after rabies virus infection. Virus Res (2009) 144(1-2):18-26. doi:10.1016/j. virusres.2009.03.014

39. Chai Q, He WQ, Zhou M, Lu H, Fu ZF. Enhancement of blood-brain barrier permeability and reduction of tight junction protein expression are modulated by chemokines/cytokines induced by rabies virus infection. J Virol (2014) 88(9):4698-710. doi:10.1128/JVI.03149-13

40. Yang Y, Huang Y, Gnanadurai CW, Cao S, Liu X, Cui M, et al. The inability of wild-type rabies virus to activate dendritic cells is dependent on the glycoprotein and correlates with its low level of the de novo-synthesized leader RNA. J Virol (2015) 89(4):2157-69. doi:10.1128/JVI.02092-14 
41. Chai Q, She R, Huang Y, Fu ZF. Expression of neuronal CXCL10 induced by rabies virus infection initiates infiltration of inflammatory cells, production of chemokines and cytokines, and enhancement of blood-brain barrier permeability. J Virol (2015) 89(1):870-6. doi:10.1128/JVI.02154-14

42. Zhao L, Rose KM, Elliott R, Van Rooijen N, Weiss SR. Cell-type-specific type I interferon antagonism influences organ tropism of murine coronavirus. J Virol (2011) 85(19):10058-68. doi:10.1128/JVI.05075-11

43. Chen CJ, Ou YC, Li JR, Chang CY, Pan HC, Lai CY, et al. Infection of pericytes in vitro by Japanese encephalitis virus disrupts the integrity of the endothelial barrier. J Virol (2014) 88(2):1150-61. doi:10.1128/JVI.02738-13

44. Daniels BP, Jujjavarapu H, Durrant DM, Williams JL, Green RR, White JP, et al. Regional astrocyte IFN signaling restricts pathogenesis during neurotropic viral infection. J Clin Invest (2017) 127(3):843-56. doi:10.1172/JCI88720

45. Gack MU, Albrecht RA, Urano T, Inn KS, Huang IC, Carnero E, et al. Influenza A virus NS1 targets the ubiquitin ligase TRIM25 to evade recognition by the host viral RNA sensor RIG-I. Cell Host Microbe (2009) 5(5):439-49. doi:10.1016/j.chom.2009.04.006

46. Faber M, Bette M, Preuss MA, Pulmanausahakul R, Rehnelt J, Schnell MJ, et al. Overexpression of tumor necrosis factor alpha by a recombinant rabies virus attenuates replication in neurons and prevents lethal infection in mice. J Virol (2005) 79(24):15405-16. doi:10.1128/JVI.79.24.15405-15416.2005

47. Nakamichi K, Saiki M, Sawada M, Takayama-Ito M, Yamamuro Y, Morimoto $\mathrm{K}$, et al. Rabies virus-induced activation of mitogen-activated protein kinase and NF-kappaB signaling pathways regulates expression of CXC and CC chemokine ligands in microglia. J Virol (2005) 79(18):11801-12. doi:10.1128/JVI.79.18.11801-11812.2005

48. Weber F, Wagner V, Rasmussen SB, Hartmann R, Paludan SR. Doublestranded RNA is produced by positive-strand RNA viruses and DNA viruses but not in detectable amounts by negative-strand RNA viruses. J Virol (2006) 80(10):5059-64. doi:10.1128/JVI.80.10.5059-5064.2006

49. Pfaller CK, Radeke MJ, Cattaneo R, Samuel CE. Measles virus C protein impairs production of defective copyback double-stranded viral RNA and activation of protein kinase R. J Virol (2014) 88(1):456-68. doi:10.1128/ JVI.02572-13

50. Peng BH, Borisevich V, Popov VL, Zacks MA, Estes DM, Campbell GA, et al. Production of IL-8, IL-17, IFN-gamma and IP-10 in human astrocytes correlates with alphavirus attenuation. Vet Microbiol (2013) 163(3-4): 223-34. doi:10.1016/j.vetmic.2012.11.021

51. Takeuchi K, Komatsu T, Kitagawa Y, Sada K, Gotoh B. Sendai virus C protein plays a role in restricting PKR activation by limiting the generation of intracellular double-stranded RNA. J Virol (2008) 82(20):10102-10. doi:10.1128/ JVI.00599-08

52. Bale S, Julien JP, Bornholdt ZA, Krois AS, Wilson IA, Saphire EO. Ebolavirus VP35 coats the backbone of double-stranded RNA for interferon antagonism. J Virol (2013) 87(18):10385-8. doi:10.1128/JVI.01452-13

53. Kindler E, Gil-Cruz C, Spanier J, Li Y, Wilhelm J, Rabouw HH, et al. Early endonuclease-mediated evasion of RNA sensing ensures efficient coronavirus replication. PLoS Pathog (2017) 13(2):e1006195. doi:10.1371/journal. ppat. 1006195

54. Lau LT, Yu AC. Astrocytes produce and release interleukin-1, interleukin-6, tumor necrosis factor alpha and interferon-gamma following traumatic and metabolic injury. J Neurotrauma (2001) 18(3):351-9. doi:10.1089/ 08977150151071035

55. Huppert J, Closhen D, Croxford A, White R, Kulig P, Pietrowski E, et al. Cellular mechanisms of IL-17-induced blood-brain barrier disruption. FASEB $J$ (2010) 24(4):1023-34. doi:10.1096/fj.09-141978

56. Barkhouse DA, Garcia SA, Bongiorno EK, Lebrun A, Faber M, Hooper DC. Expression of interferon gamma by a recombinant rabies virus strongly attenuates the pathogenicity of the virus via induction of type I interferon. J Virol (2015) 89(1):312-22. doi:10.1128/JVI.01572-14

Conflict of Interest Statement: The authors declare that the research was conducted in the absence of any commercial or financial relationships that could be as a potential conflict of interest.

Copyright (c) 2018 Tian, Zhou, Yang, Yu, Luo, Tian, Wang, Cui, Chen, Fu and Zhao. This is an open-access article distributed under the terms of the Creative Commons Attribution License (CC BY). The use, distribution or reproduction in other forums is permitted, provided the original author(s) or licensor are credited and that the original publication in this journal is cited, in accordance with accepted academic practice. No use, distribution or reproduction is permitted which does not comply with these terms. 